\title{
Ophthalmic Glucose Monitoring Using Disposable Contact Lenses-A Review
}

\author{
Ramachandram Badugu, ${ }^{1}$ Joseph R. Lakowicz, ${ }^{1,3}$ and Chris D. Geddes ${ }^{1,2,3}$
}

Received

\begin{abstract}
We have developed a range of disposable and colorless tear glucose sensing contact lenses, using offthe-shelf lenses embedded with new water soluble, highly fluorescent and glucose sensitive boronic acid containing fluorophores. The new lenses are readily able to track tear glucose levels and therefore blood glucose levels, which are ideally suited for potential use by diabetics. The fluorescence responses from the lenses can be monitored using simple excitation and emission detection devices. The novelty of our approach is two fold. Firstly, the notion of sensing extremely low glucose concentrations in tears, which track blood levels, by our contact lens approach, and secondly, the unique compatibility of our new glucose signaling probes with the internal mildly acidic contact lens environment. The new lenses are therefore ideal for the non-invasive and continuous monitoring of tear glucose, with about 15-min response time, and a measured shelf life in excess of 3 months. In this review article, we show that fluorescence based signaling using plastic disposable lenses, which have already been industrially optimized with regard to vision correction and oxygen/analyte permeability etc, may a notable alternative to invasive and random finger pricking, the most widely used glucose monitoring technology by diabetics.
\end{abstract}

KEY WORDS: Ophthalmic glucose monitoring; tear glucose; glucose sensing; monosaccharides, contact lens; non-invasive sensing; continuous glucose monitoring; clinical assessment; drug compliance; diabetes.

\section{INTRODUCTION}

As a common medical condition that produces excessive thirst, continuous urination and severe weight loss, Diabetes ${ }^{4}$ has interested medical researchers for over three millennia. Unfortunately it wasn't until the early 20th cen-

\footnotetext{
${ }^{1}$ Department of Biochemistry and Molecular Biology, Center for Fluorescence Spectroscopy, Medical Biotechnology Center, University of Maryland School of Medicine, Baltimore, Maryland.

${ }^{2}$ Institute of Fluorescence, Medical Biotechnology Center, University of Maryland Biotechnology Institute, Baltimore, Maryland.

${ }^{3}$ To whom correspondence should be addressed at Department of Biochemistry and Molecular Biology, Center for Fluorescence Spectroscopy, Medical Biotechnology Center, University of Maryland School of Medicine, 725 West Lombard Street, Baltimore, Maryland 21201. E-mail: geddes@umbi.umd.edu or lakowicz@cfs.umbi.umd. edu

${ }^{4}$ The term "Diabetes" was first used around $230 \mathrm{BC}$ by Apollonius of Mephis, which in Greek means "to pass through" (Dia-through, betes - to go) [1].
}

tury that the prognosis for this condition became any better than it was 3000 years ago. Today, approximately 150 million people worldwide are affected by diabetes. With its prevalence still rising, diabetes still continues to fascinate, practitioners and researchers alike by its elusive cause and its many manifestations.

While the Ebers Papyrus, which was written around $1500 \mathrm{BC}$, excavated in $1862 \mathrm{AD}$ from an ancient grave in Thebes, Egypt, described the first reference to Diabetes Mellitus, it was physicians in India at around the same

\footnotetext{
Abbreviations: BA, Boronic Acid; BAFs, Boronic acid containing fluorophores; BMQ, $N$-Benzyl-6-methylquinolinium bromide; BMOQ, $N$-Benzyl-6-methoxyquinolinium bromide; BMQBA, $N$-(Boronobenzyl)- 6-methylquinolinium bromide; BMOQBA, $N$-(Boronobenzyl)-6-methoxyquinolinium bromide; CT, Excited-state Charge Transfer; DDPBBA, 1-( $p$-Boronophenyl)-4-( $p$-dimethylaminophenyl)buta-1,2-deine; HRMS, High-Resolution Mass Spectroscopy; LD, Laser Diode; LED, Light Emitting Diode; PVA, Polyvinyl alcohol; TCSPC, Time-correlated Single Photon Counting.
} 
time that developed the first crude test for diabetes. They observed that the urine from people with diabetes attracted ants and flies. They subsequently named the condition "madhumeha" or "honey urine" [1].

The important elements of our current understanding of diabetes can be traced to the early to mid 19th century. In 1815 Eugene Chevreul in Paris concluded that the sugar in urine was indeed Glucose, the first quantitative test for glucose in urine being developed by Von Fehling some years later in 1848 [2].

Over 150 years later, significant attention is still given to the development of physiological glucose monitoring [3-36]. This is because one important aspect for diabetes management, involves the tight control of blood glucose levels, so as to manage food intake and the dosage and timing of insulin injection. Tests for determining serum glucose concentration typically require blood collection by some invasive technique, usually a needle or other device causing arterial or venous puncture. Currently, millions of diabetics are left with very few alternatives, except to invasively draw blood many times daily to determine their blood sugar levels. To this end, many technologies have been developed over the past 20 years in an attempt to provide a technology which promises both non-invasive and continuous physiological glucose monitoring. These include near infrared spectroscopy $[3,4]$, optical rotation $[5,6]$, colorimetric $[7,8]$ and fluorescence detection [913], to name but just a very few. Recently we have seen the launch of the new GlucoWatch, which approved by the FDA in 2001, is the first step towards both the continuous and non-invasive monitoring of physiological glucose. However, in addition to wearing this wrist watch based glucose sensor, it is also recommended that glucose monitoring by another blood sampling technique be additionally used from time to time. Other technologies which are presently emerging include, Glucose monitoring skin Patches, implantable glucose sensors coupled insulin pumps, and laser blood drawing, which is deemed less painful than finger pricking with a lancet or needle. Yet with all these emerging technologies, there is still a need for new technologies, which are truly non-invasive and continuous. To this end our laboratories have been developing glucose sensing contact lenses which when worn by diabetics, who often require vision correction in any case, can potentially monitor tear glucose levels, which are known to directly relate and track blood glucose levels [14-17].

In this review article of work from our laboratories, we employ the notion of elevated tear glucose levels during hyperglycemia to investigate, for the first time, the possibility of monitoring tear glucose and therefore blood glucose, using a disposable, off-the-shelf, contact lens.

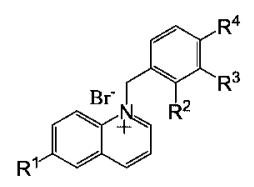

\begin{tabular}{llccc}
\hline Probe & $\mathbf{R}^{\mathbf{1}}$ & $\mathbf{R}^{\mathbf{2}}$ & $\mathbf{R}^{\mathbf{3}}$ & $\mathbf{R}^{\mathbf{4}}$ \\
\hline $\boldsymbol{o}$-BMOQBA & $\mathrm{OCH}_{3}$ & $\mathrm{~B}(\mathrm{OH})_{2}$ & $\mathrm{H}$ & $\mathrm{H}$ \\
$m$-BMOQBA & $\mathrm{OCH}_{3}$ & $\mathrm{H}$ & $\mathrm{B}(\mathrm{OH})_{2}$ & $\mathrm{H}$ \\
$\boldsymbol{p}$-BMOQBA & $\mathrm{OCH}_{3}$ & $\mathrm{H}$ & $\mathrm{H}$ & $\mathrm{B}(\mathrm{OH})_{2}$ \\
BMOQ & $\mathrm{OCH}_{3}$ & $\mathrm{H}$ & $\mathrm{H}$ & $\mathrm{H}$ \\
\hline $\boldsymbol{o}$-BMQBA & $\mathrm{CH}_{3}$ & $\mathrm{~B}(\mathrm{OH})_{2}$ & $\mathrm{H}$ & $\mathrm{H}$ \\
$m$-BMQBA & $\mathrm{CH}_{3}$ & $\mathrm{H}$ & $\mathrm{B}(\mathrm{OH})_{2}$ & $\mathrm{H}$ \\
$\boldsymbol{p}$-BMQBA & $\mathrm{CH}_{3}$ & $\mathrm{H}$ & $\mathrm{H}$ & $\mathrm{B}(\mathrm{OH})_{2}$ \\
BMQ & $\mathrm{CH}_{3}$ & $\mathrm{H}$ & $\mathbf{H}$ & $\mathrm{H}$ \\
\hline
\end{tabular}

Fig. 1. Molecular structure of the boronic acid probes, ortho-, metaand para-BMOQBA, BMQBAs, and the respective control compounds BMOQ and BMQ. BMOQBA: $N$-(boronobenzyl)-6-methoxyquinolinium bromide, BMOQ: $N$-benzyl-6-methoxyquinolinium bromide. BMQBA: $N$-(boronobenzyl)-6-|methylquinolinium bromide, BMQ: $N$-benzyl-6-methylquinolinium bromide.

By incorporating new monosaccharide signaling fluorescent probes within such a lens (Fig. 1), we can indeed make progress towards this non-invasive and continuous approach for glucose monitoring (Fig. 2).

As with any sensors, there are several issues that have to be addressed. The first is to identify suitable transduction elements, which in the presence of glucose, can report/produce suitable signals. The second is the design of the matrix to incorporate the transduction elements. For this, we have chosen an off-the-shelf disposable plastic contact lens, primarily because its physiological compatibility has already been assessed, and finally, the optimization of the sensor, with regard to sensitivity, response time, reversibility and shelf-life etc. The later two issues will be discussed throughout much of this review and indeed in past papers by the authors [14-17]. For the identification of suitable transduction elements, boronic acid has been known to have high affinity for diol-containing compounds such as carbohydrates [18-20] (Scheme 1), where the strong complexation has been used for the construction of carbohydrate sensors [21-28], transporters [29] and chromatographic materials [30]. Boronic acid compounds have also been used for the synthesis of glucose sensors [31-36], where we note the work of Shinkai [31,32] and Lakowicz [33-36] to name but just a few workers in this field.

Boronic acids are weak Lewis Acids composed of an electron deficient boron atom and two hydroxyl groups, (1 in scheme 1), which can interact with strong bases like 

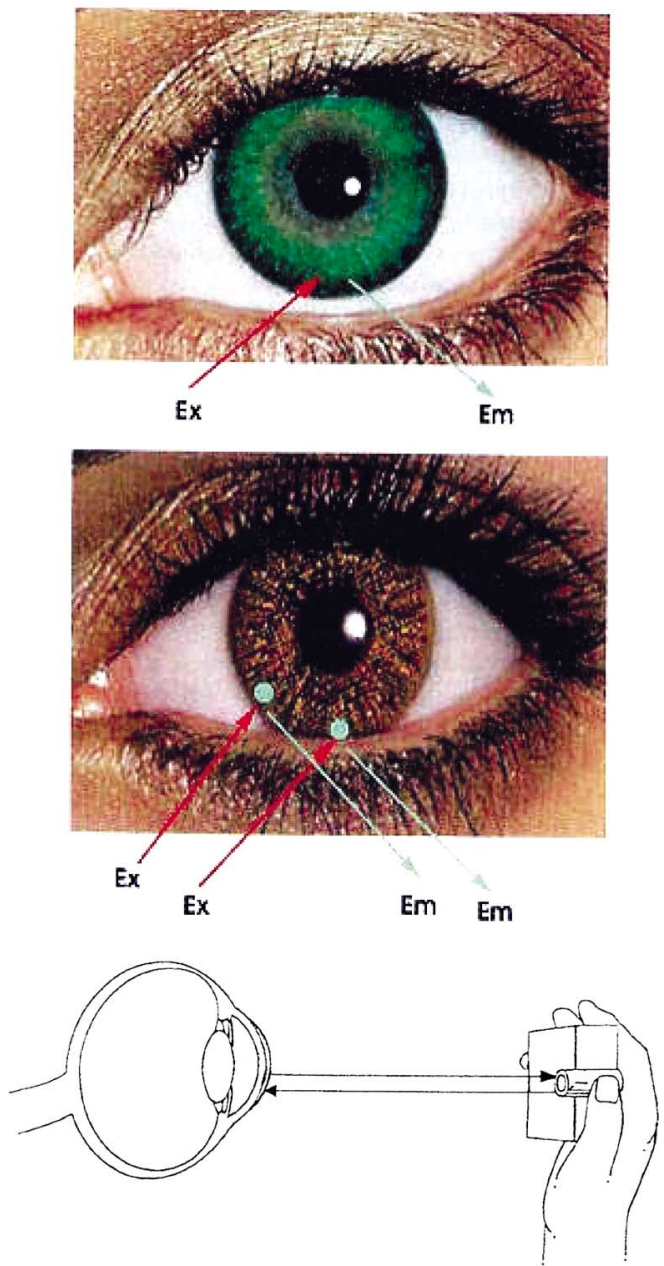

Fig. 2. Potential methods for non-invasive continuous tear glucose monitoring. (Top) BAF doped contact lens as described here, and (Middle) Sensor spots on the surface of the lens to additionally monitor other analytes in addition to glucose, such as drugs, biological markers, $\mathrm{Ca}^{2+}, \mathrm{K}^{+}, \mathrm{Na}^{+}, \mathrm{O}_{2}$ and $\mathrm{Cl}^{-}$. Sensor regions may also allow for ratiometric, lifetime or polarization based fluorescence glucose sensing. (Bottom) Schematic representation of the possible tear glucose sensing device.

$\mathrm{OH}^{-}$to from the anionic borate form (2 in scheme 1), showing typically high $\mathrm{p} K_{\mathrm{a}}$ around 9 [37,38]. Boronic acids couple with diols to form a boronic acid diester group ( 3 in scheme 1). The diol is linked covalently, and the reaction is fast and completely reversible [38]. In comparison to the boronic acid group, the boronic acid ester group shows higher acidity $\left(\mathrm{p} K_{\mathrm{a}} \approx 6\right)$ due to a higher electrophilic boron atom. The monophenylboronic acid group shows higher affinity for D-fructose with a smaller affinity for D-glucose [38], with binding constants of $\approx$ 0.5 and $10 \mathrm{mM}$ respectively [38]. The use of the boronic acid groups for sensing sugars is strongly dependent on

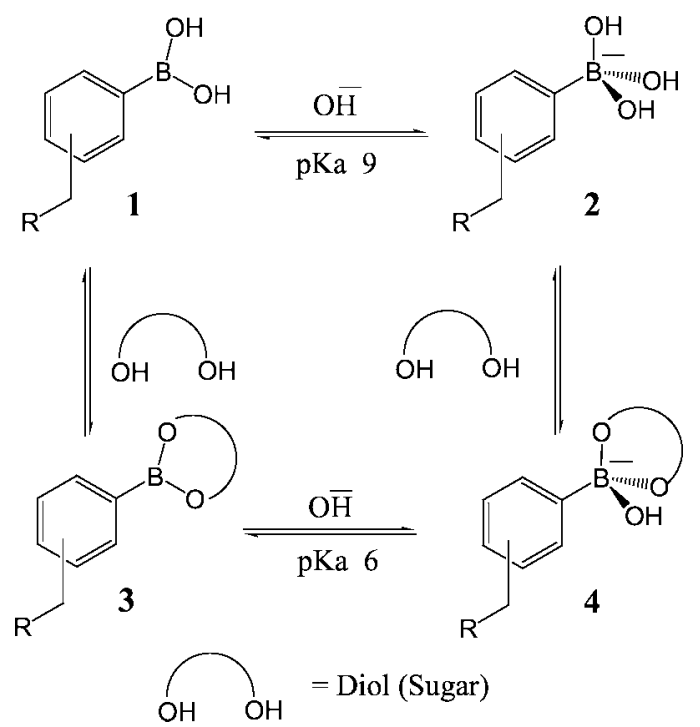

Scheme 1. Equilibrium for the boronic acid/diol (sugar) interaction.

the molecular geometry and the aromatic species where the boronic acid group is present, hence glucose sensitive probes can be made with a variety of affinities, in the $\mathrm{mM}$ range for blood glucose [34-36], and in the $\mu \mathrm{M}$ range for tear glucose $[39,40]$.

\section{LENS FEASIBILITY STUDY}

To determine the usefulness of glucose signaling probes based on the boronic acid glucose chelating moiety, we initially compared the response towards glucose of a wide variety of published BAFs when doped within the contact lenses [14].

In most cases the response towards glucose in the lenses was significantly different, with drastically reduced glucose responses [14]. Figure 3 shows the response of one boronic acid containing fluorophore, DDPBBA, towards glucose within the lens, as compared to the response typically observed at physiological pH (Fig. 4). As with the other probes studied, we observed that the response towards glucose in the contact lens was similar to the response observed in $\approx \mathrm{pH} 6.0$ bulk solution (cf. Figs. 3 and 4 bottom right). We subsequently doped the well-known $\mathrm{pH}$ sensitive probe fluorescein [41] within the lenses, and determined that the lenses had an unbufferable lens $\mathrm{pH}$ of $\approx 6.1$ (Fig. 5 top). However, the influence of an acidic lens $\mathrm{pH}$ does not solely explain the spectral shifts observed in Figs. 3 and 4, with DDPBBA additionally showing a $50 \mathrm{~nm}$ hyposchromic shift. An investigation of the local polarity within the contact lens, using pyrene 

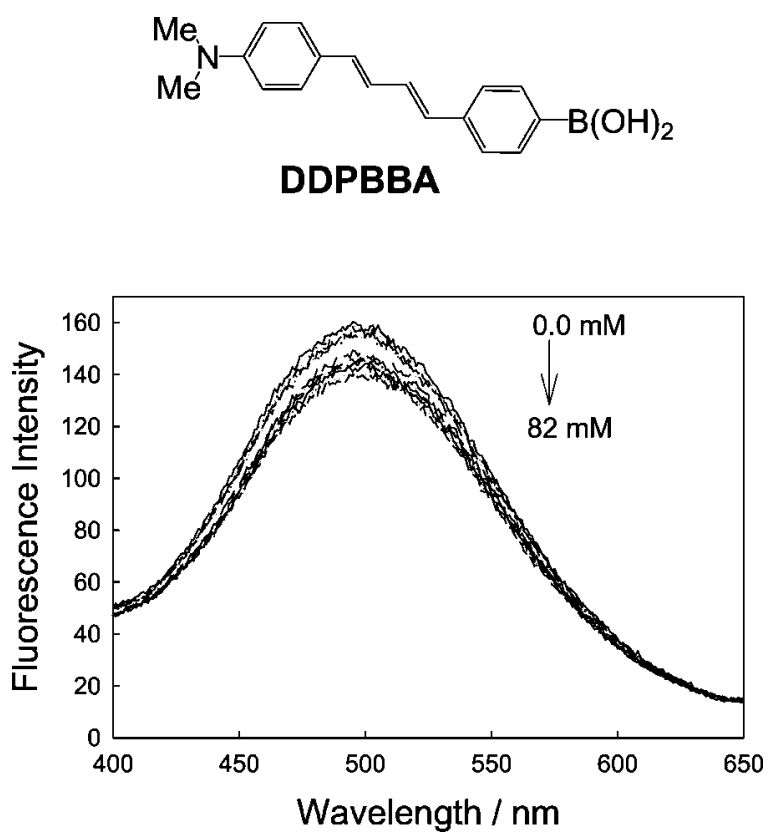

Fig. 3. Molecular structures of the $C T$ probe studied in the contact lens (Top). DDPBBA-1-( $p$-Boronophenyl)-4-( $p$-dimethylaminophenyl)buta-1,2-deine. Emission spectra of the DDPBBA doped contact lens, $\mathrm{pH} 8.0$ buffer/methanol (2:1), with increasing concentrations of glucose, $\lambda_{\mathrm{ex}}=340 \mathrm{~nm}$ (Bottom).
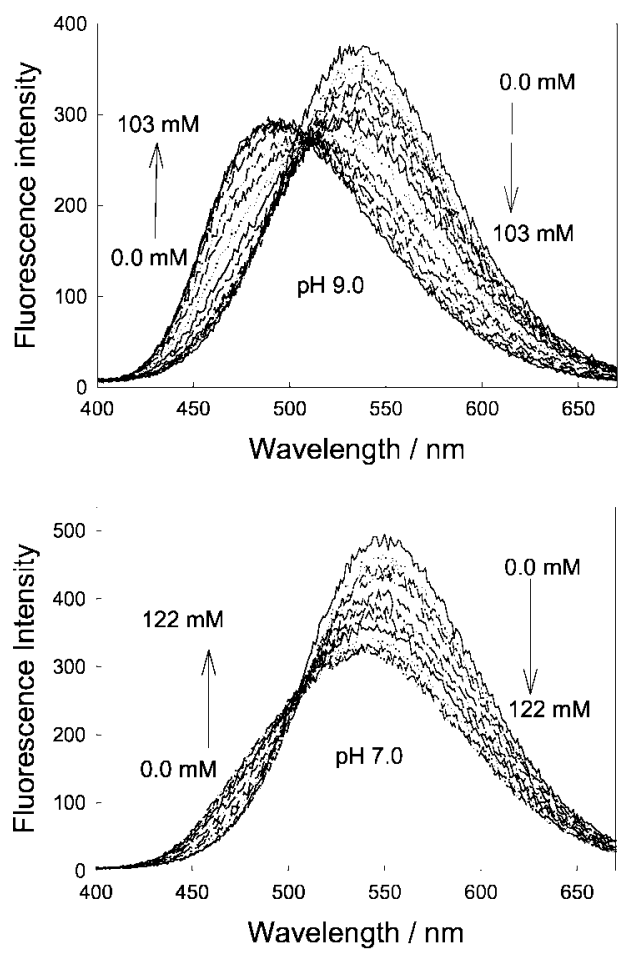

Fig. 4. Emission spectra of DDPBBA in different $\mathrm{pH}$ media (buffer/methanol 2:1) with increasing glucose concentrations. Adapted from [14].
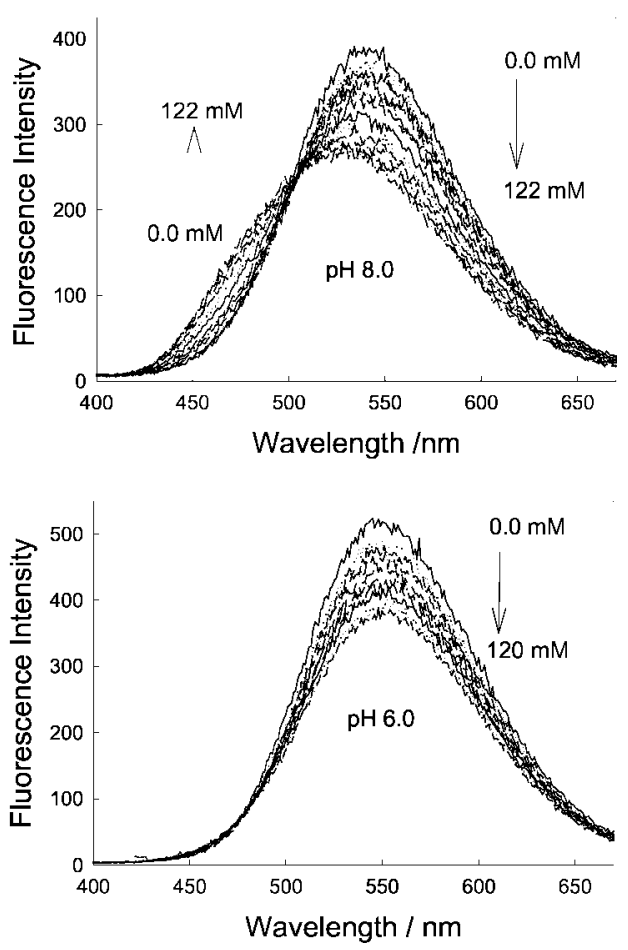

$[14,42,43]$ (Fig. 5 bottom), revealed a lens polarity similar to that of methanol, which in hindsight was not too surprising, given the nature of the polymer, i.e. it is PVA based.

\section{RATIONALE FOR THE DESIGN OF NEW GLUCOSE SIGNALING PROBES}

Feasibility studies of doped lenses with published boronic acid containing fluorophores had produced poor glucose responses, rationaled as due to the mildly acidic $\mathrm{pH}$ and methanol like polarity within the lens and the subsequent effect on the CT mechanism. This was also not surprising given the fact that these probes were designed for sensing at a physiological $\mathrm{pH}$ of $\approx 7.2$, the probes typically having $\mathrm{p} K_{\mathrm{a}}$ around 9 . Hence to obtain a notable glucose response in the contact lens polymer, it was deemed necessary to design new probes with significantly reduced sugar-bound $\mathrm{p} K_{\mathrm{a}}$. In addition to the environmental parameters and constraints of $\mathrm{pH}$ and polarity, the probes also have to be sensitive to the very low concentrations of tear glucose, $\approx 500 \mu \mathrm{M}$ for a healthy person, increasing up to several $\mathrm{mM}$ in diabetics, recalling that the blood glucose levels for a healthy person are $\approx 10$-fold higher [14-17]. 

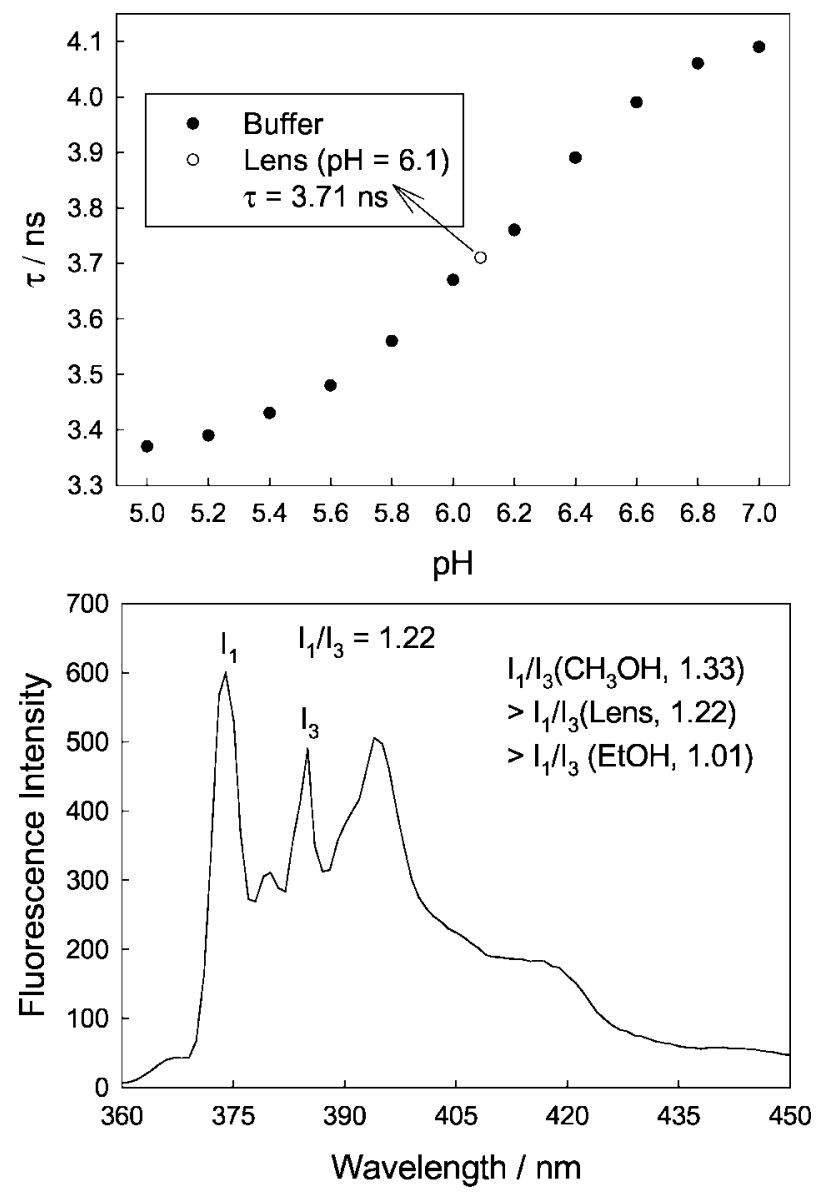

Fig. 5. The calibration plot of fluorescein lifetime with increasing $\mathrm{pH}$, and the subsequent $\mathrm{pH}$ value in the lens (Top). The pyrene fluorescence spectra in a contact lens $\left(l_{\mathrm{ex}}=340 \mathrm{~nm}\right)$ measured to obtain the polarity inside the lens.

The $\mathrm{p} K_{\mathrm{a}}$ of phenyl boronic acid is known to be tunable with the appropriate substituents [44], for example, an electron withdrawing group reduces the $\mathrm{p} K_{\mathrm{a}}$ while an electron donating group increases the $\mathrm{p} K_{\mathrm{a}}$ of the sugar bound form. We therefore considered the interaction between the quaternary nitrogen of the 6-methyl and methoxy quinolinium moieties, and the boronic acid group, which reduces the $\mathrm{p} K_{\mathrm{a}}$ of the probe. In this regard we have synthesized 2 new classes of isomeric boronic acid containing probes (8-probes in total) (Fig. 1), where the spacing between the interacting moieties, quaternary nitrogen of the 6-methyl or methoxy quinolinium and boronic acid groups, enables both an understanding of the sensing mechanism to be realized, and the selection of the most suitable isomer based on its glucose binding affinity. In addition, control compounds (BMQ and BMOQ), which do not contain the boronic acid moiety, and are therefore insensitive towards sugar, were synthesized to understand the spectral properties and responses of the probes (Fig. 1).

\section{EXPERIMENTAL}

\section{Materials and Lens Doping}

All chemicals were purchased from Sigma. The contact lenses were supplied by CIBA Vision, Atlanta, USA, part of their daily disposable contact lens range, and were stirred in $500 \mathrm{~mL}$ water, $20^{\circ} \mathrm{C}$ for $24 \mathrm{hr}$ before post-doping. The contact lens is a Polyvinyl alcohol type photocured polymer which swells slightly in water. Its hydrophilic character readily allows for the diffusion of the aqueous analytes in tears.

Doping was undertaken by incubating the lenses in a high concentration of the respective BAFs solution for $24 \mathrm{hr}$ before being rinsed in Millipore water. Lenses were used directly after being prepared.

The preparation of the BAFs (Fig. 1) was undertaken as briefly follows, and are described in more detail elsewhere $[39,40]$.

\section{Methyl Quinolinium BAFs}

The Boronic acid containing probes (o-BMQBA$N$-(2-boronobenzyl)-6-methylquinolinium bromide, $m$-BMQBA- $N$-(3-boronobenzyl)-6-methylquinolinium bromide, $p$-BMQBA- $N$-(4-boronobenzyl)-6-methylquinolinium bromide) and the control compound (BMQ$N$-benzyl-6-methylquinolinium bromide), were conveniently prepared using the following generic one step synthetic procedure, described below for the control compound BMQ. The corresponding $o-, m-$, or $p$ boronobenzyl bromides are employed instead of benzyl bromide to obtain the isomeric boronic acid derivatives $o-, m$ - and $p$-BMQBA, respectively (Fig. 1).

Equimolar amounts of 6-methylquinoline and benzylbromide were dissolved in $10 \mathrm{~mL}$ dry acetonitrile in a $25 \mathrm{~mL}$ round bottomed flask equipped with a magnetic stirrer. The reaction mixture was allowed to stir under an inert atmosphere for $24 \mathrm{hr}$ at room temperature. During this time a quantitative amount of quaternized salt was precipitated as a colorless solid. The solid product was recovered by filtration, washed several times with dry acetonitrile, and then dried under vacuum for $12 \mathrm{hr}$. The NMR and HRMS data obtained for all 8 compounds was consistence with the structure of the probes. 


\section{Methoxy Quinolinium BAFs}

The glucose signaling probes based on the 6-methoxy quinoline nucleus; $o-, m$ - and $p$-BMOQBA and a control compound BMOQ (Fig. 1) were prepared in an analogous manner to the methyl quinolinium probes described above, using 6-methoxy quinoline instead of 6-methyl quinoline as the starting reagent.

\section{The Contact Lens Holder}

The quartz lens holder which was used in all the lens studies, has dimensions of $4 \times 2.5 \times 0.8 \mathrm{~cm}$, all four sides being of optical quality. The contact lens is mounted onto a stainless steel mount of dimensions $4 \times$ $2 \times 0.4 \mathrm{~cm}$ which fits tightly within the quartz outer holder. A circular hole in the center of the mount with a $2.5 \mathrm{~cm} \mathrm{ID}$, has a raised quartz lip, which enables the lens to be mounted. The mount and holder readily allow for $\approx$ $1.5 \mathrm{~cm}^{3}$ of solution to be in contact with the front and back sides of the lens for the sugar sensing experiments (Fig. 6 left).

\section{Methods}

All steady-state fluorescence measurements were undertaken in $4 \times 1 \times 1 \mathrm{~cm}$ fluorometric plastic cuvettes, using a Varian Cary Eclipse fluorometer, and all absorption measurements were performed using a Varian UV/VIS 50 spectrophotometer.

Time-resolved intensity decays were measured using reverse start-stop time-correlated single-photon timing (TCSPC), with a Becker and Hickl Gmbh 630 SPCPC card and unamplified MCP-PMT. Vertically polarized excitation at $\approx 372 \mathrm{~nm}$ was obtained using a pulsed LED source (1 $\mathrm{MHz}$ repetition rate) and a dichroic sheet polarizer. The instrumental response function was $\approx 1.1 \mathrm{~ns}$ fwhm. The emission was collected at the magic angle $\left(54.7^{\circ}\right)$, using a long pass filter (Edmund Scientific) which cut-off the excitation wavelengths.

Doped contact lenses were mounted in a custom made (CIBA Vision) lens holder (Fig. 6), which was itself inserted into a quartz holder for fluorescence sensing measurements. Excitation and emission was performed using a Varian Fluorometer, where the geometry shown in Fig. 6 (right), was employed to reduce any scattering of the excitation light, the concave edge of the lens facing towards the excitation source. We additionally tested the lens excited from the convex edge, just as would be used in the eye, and encouragingly found identical results.

\section{Probe Leaching}

Leaching of the probes from the contact lens polymer was observed using the sample holder shown in Fig. 6 , which contained $\approx 1.5 \mathrm{~cm}^{3}$ buffer, $20^{\circ} \mathrm{C}$. A Varian

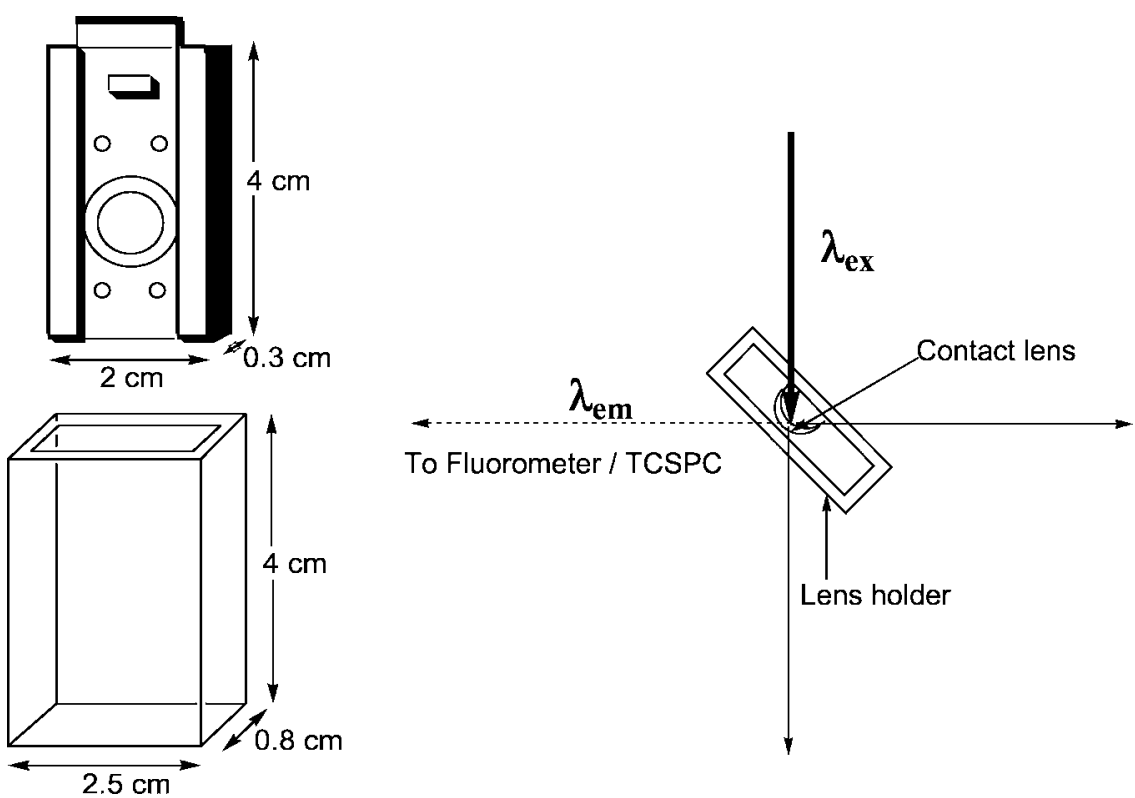

Fig. 6. Contact lens mount and quartz holder (Left) and experimental geometry used for contact lens glucose sensing (Right). Adapted from [14]. 
fluorometer measured the intensity change as a function of time to determine the percentage signal change, corresponding to dye leaching. It should be noted that with no sample present, no intensity fluctuations or drifts were observed, indicating stability of the fluorometer Xenonarc source.

\section{Data Analysis}

Titration curves with $\mathrm{pH}$ were determined in buffer solution: $\mathrm{pH} 3$ and 4 acetate buffer; $\mathrm{pH}$ 5-9 phosphate buffer and $\mathrm{pH} 10$ and 11 carbonate buffer. Titration curves were fitted and $\mathrm{p} K_{\mathrm{a}}\left(\mathrm{p} K_{\mathrm{a}}=-\log _{10} K_{a}\right)$ values were obtained using the relation:

$$
I=\frac{10^{-\mathrm{pH}} I_{\mathrm{acid}}+K_{a} I_{\text {base }}}{K_{a}+10^{-\mathrm{pH}}}
$$

where $I_{\text {acid }}$ and $I_{\text {base }}$ are the intensity limits in the acid and base regions respectively.

Stability $\left(K_{\mathrm{S}}\right)$ and dissociation $\left(K_{\mathrm{D}}\right)$ constants were obtained by fitting the titration curves, with sugar, using the relation:

$$
I=\frac{I_{\min }+I_{\max } K_{\mathrm{S}}[\text { sugar }]}{1+K_{\mathrm{S}}[\text { sugar }]}
$$

where $I_{\min }$ and $I_{\max }$ are the initial (no sugar) and final (plateau) fluorescence intensities of the titration curves, where $K_{\mathrm{D}}=\left(1 / K_{S}\right)$.

The fluorescence intensity decays were analyzed in terms of the multi-exponential model:

$$
I(t)=\sum_{i} \alpha_{i} \exp \left(-t / \tau_{i}\right)
$$

where $\alpha_{I}$ are the amplitudes and $\tau_{I}$ the decay times, $\Sigma \alpha_{I}=$ 1.0. The fractional contribution of each component to the steady-state intensity is given by:

$$
f_{i}=\frac{\alpha_{i} \tau_{i}}{\sum_{i} \alpha_{i} \tau_{i}}
$$

The mean lifetime of the excited state is given by:

$$
\bar{\tau}=\sum_{i} f_{i} \tau_{i}
$$

and the amplitude-weighted lifetime is given by:

$$
\langle\tau\rangle=\sum_{i} \alpha_{i} \tau_{i}
$$

The values of $\alpha_{I}$ and $\tau_{I}$ were determined by non-linear least squares impulse reconvolution with a goodness-offit $\chi_{R}^{2}$ criterion [41].

\section{RESULTS AND DISCUSSION}

\section{Photophysical Characterization of the Quinolinium Probes}

Figure 7 shows representative absorption and emission spectra for the ortho-isomers of the BMOQBA and BMQBA probes. Photophysical data of the probes are also shown in Table I. Typical absorption and emission band maximum of the probes can be seen at 318 and $450 \mathrm{~nm}$, and 319 and $427 \mathrm{~nm}$ for the BMOQBA and BMQBA probes respectively. The additional absorption band at $\approx 350 \mathrm{~nm}$ for BMOQBA is attributed to the $n \rightarrow \pi^{*}$ transition of the oxygen [45]. The excitation independent emission band at $\approx 450 \mathrm{~nm}$ indicates only one ground-state species is present for both classes of probes. The large Stokesshifted fluorescence emission band of $\approx 100 \mathrm{~nm}$ is ideal for fluorescence sensing, allowing easy discrimination of
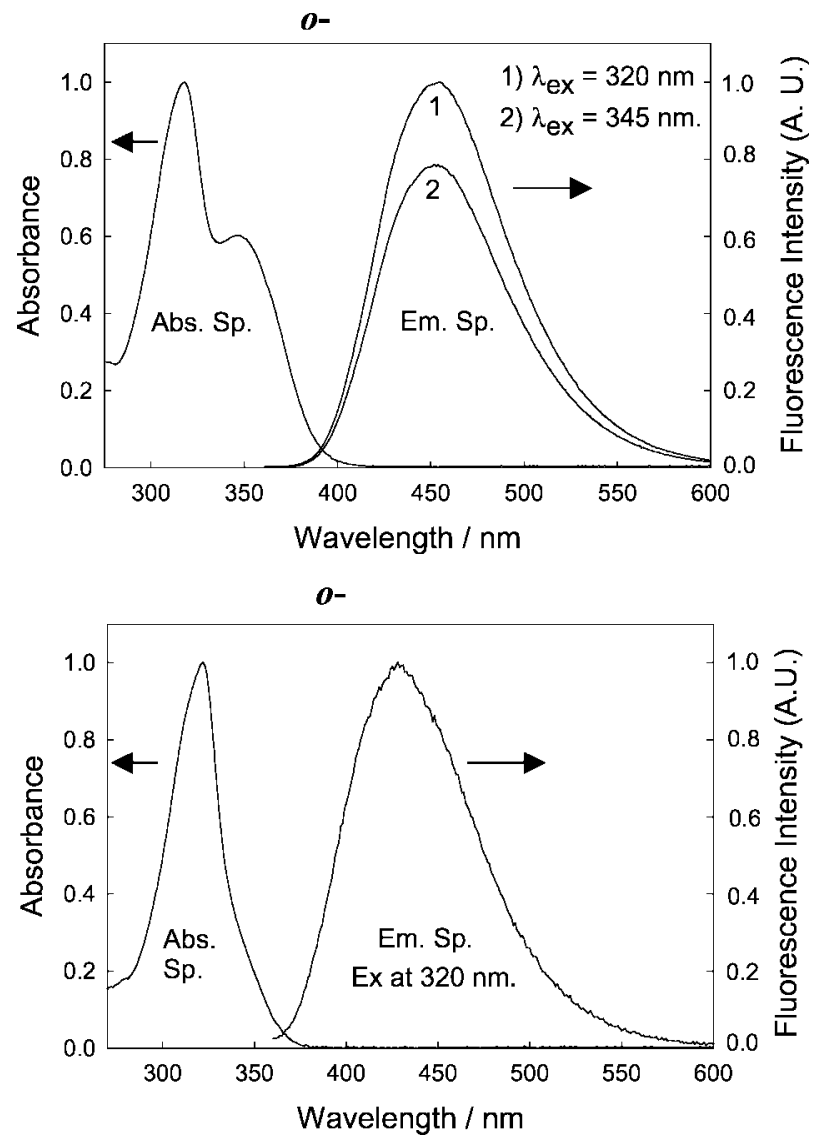

Fig. 7. Absorption and emission spectra of $o$-BMOQBA (Top) and $o$ BMQBA (Bottom) in water. The spectra are representative of the respective isomeric phenylboronic acid containing fluorophores and the control compounds. 
Table I. Photophysical Data of the Probes in Water at Room Temperature

\begin{tabular}{lcccc}
\hline & $o$-BMOQBA & $m$-BMOQBA & $p$-BMOQBA & BMOQ \\
\hline$\lambda_{\text {abs }}(\max ) / \mathrm{nm}$ & 318,346 & 318,347 & 318,346 & 318,347 \\
$\lambda_{\text {em }}(\max ) / \mathrm{nm}$ & 450 & 450 & 451 & 453 \\
$\phi_{f}$ & 0.46 & 0.51 & 0.49 & 0.54 \\
$\tau_{f} / \mathrm{ns}$ & 26.7 & 25.9 & 24.9 & 27.3 \\
& $o$-BMQBA & $m$-BMQBA & $p$-BMQBA & BMQ \\
\hline$\lambda_{\text {abs }}(\max ) / \mathrm{nm}$ & 319 & 322 & 322 & 322 \\
$\lambda_{\text {em }}(\max ) / \mathrm{nm}$ & 427 & 427 & 427 & 427 \\
$\phi_{f}$ & 0.043 & 0.025 & 0.023 & 0.045 \\
$\tau_{f} / \mathrm{ns}$ & $4.0^{a}$ & $3.72^{a}$ & $2.10^{a}$ & 2.59 \\
\hline
\end{tabular}

${ }^{a}$ Mean fluorescence lifetime, Eq. 5 .

the excitation wavelengths $[41,45]$. All probes were found to be readily water soluble.

Table I shows the quantum yield values for the probes in water, obtained from a spectral comparison with $N$-(3-Sulfopropyl)-6-methoxyquinolinium [(SPQ) $\left(\Phi_{f}=0.53\right.$ in water [46])], where we can see that the BMOQBA probes have significantly larger quantum yields as compared to the BMQBA probes. Another reference compound, $N$-methyl-6-methylquinolinium bromide (MMQ) previously published by the authors [47] exhibits very similar spectral properties, except for a noticeable quantum yield and mean lifetime difference, approximately 10 -fold higher than its methyl quinolinium counterpart, namely BMQBA. This indicates an interaction between the phenyl ring and quinolinium moiety for the BMQBA and BMQ probes, Table I, which is not present, or present to a much lesser extent, for the BMOQBA probes. We have attributed the relatively shorter lifetime and quantum yields of the new BMQBA probes and control compound to a photo-induced electron transfer mechanism, where the phenyl ring of BMQBA is the donor, and the quaternary nitrogen heterocyclic center is the acceptor. On this note, the BMOQBA probes were found to have monoexponential lifetimes $(\approx 24.9 \rightarrow$ $26.7 \mathrm{~ns}$ ) as compared to the BMQBA probes which were biexponential in water, with significantly reduced lifetimes of 2.18 (46\% amplitude) and $4.74 \mathrm{~ns}$ (54\% amplitude), for the ortho-isomer. Both control compounds were found to have monoexponential lifetimes in water, with the BMOQ over ten times longer, $27.3 \mathrm{~ns}$, as compared to $\mathrm{BMQ}, 2.59 \mathrm{~ns}$. These lifetime changes further support the electron transfer hypothesis, the $\mathrm{B}^{-}(\mathrm{OH})_{3}$ present at neutral $\mathrm{pH}$ further reducing the lifetime of the boronic acid probes, i.e. 2 in scheme 1.

In addition to the quantum yield and fluorescence lifetime differences between the phenyl ring containing
BMQBA and BMQ probes, and the MMQ probe, we can also see lifetime differences between the BMQBA isomers themselves. We have attributed these changes due to the changes in electron donating ability of the different phenyl isomers, and additionally to their different through-space/through-bond interactions $[48,49]$ with the positively charged quaternary nitrogen center, noting again that some $\mathrm{B}^{-}(\mathrm{OH})_{3}$ is likely to be present at neutral $\mathrm{pH}$.

The quantum yield values of the BMOQBA isomers are slightly lower than that of the control compound (BMOQ), the quantum yield values increasing in the order ortho-, para- and meta-. In contrast the monoexponential fluorescence lifetimes of the isomers increased in the order para-, meta- and then ortho-, which was slightly surprising as the quantum yields and lifetimes usually change in unison. Similar to having the highest quantum yield, the control compound also had as expected, the longest lifetime, some $27.3 \mathrm{~ns}$. One explanation for these differences between the isomers, lies in the interaction between the boronate ester form present in solution, $\mathrm{B}^{-}(\mathrm{OH})_{3}, 2$ in Scheme 1, and the positively charged nitrogen center at neutral $\mathrm{pH}$, the extent of which being determined by the isomer spacing.

The emission spectra of the new boronic acid containing probes, as the $\mathrm{pH}$ increases from 3 to 11 , typically show a steady decrease in fluorescence intensity (data not shown). In contrast the control compounds, BMOQ and $\mathrm{BMQ}$, having no boronic acid group, shows no change in fluorescence intensity. Subsequently the corresponding titration curves in the absence and in the presence of $100 \mathrm{mM}$ glucose and fructose, obtained by plotting the normalized intensities at band maximum versus $\mathrm{pH}$, are shown in Fig. 8.

The $\mathrm{p} K_{\mathrm{a}}$ values obtained using Eq. 1 and the titration curves shown in Fig. 8 are shown in Table II. We can see considerably reduced $\mathrm{p} K_{\mathrm{a}}$ values for the new phenylboronic acid containing fluorophores in buffered media, as compared to the typical boronic acid probes reported in the literature [31-36], which are in the range 8-9. The quaternary nitrogen of the quinolinium nucleus not only reduces the $\mathrm{p} K_{\mathrm{a}}$ of the probes, but also serves to stabilize the boronatediester, formed upon sugar complexation. This in turn increases the affinity of the probes for sugar as shown in Table III. Hence the reduced sugar bound $\mathrm{p} K_{\mathrm{a}}$ of these new probes, coupled with their increased glucose affinity, is most attractive for our glucose sensing contact lens application, noting our previous findings of a lens $\mathrm{pH}$ around 6.1 [14].

To understand this new fluorescence signaling base mechanism and the response of the probes towards both $\mathrm{pH}$ and monosaccharides, it is informative to consider 

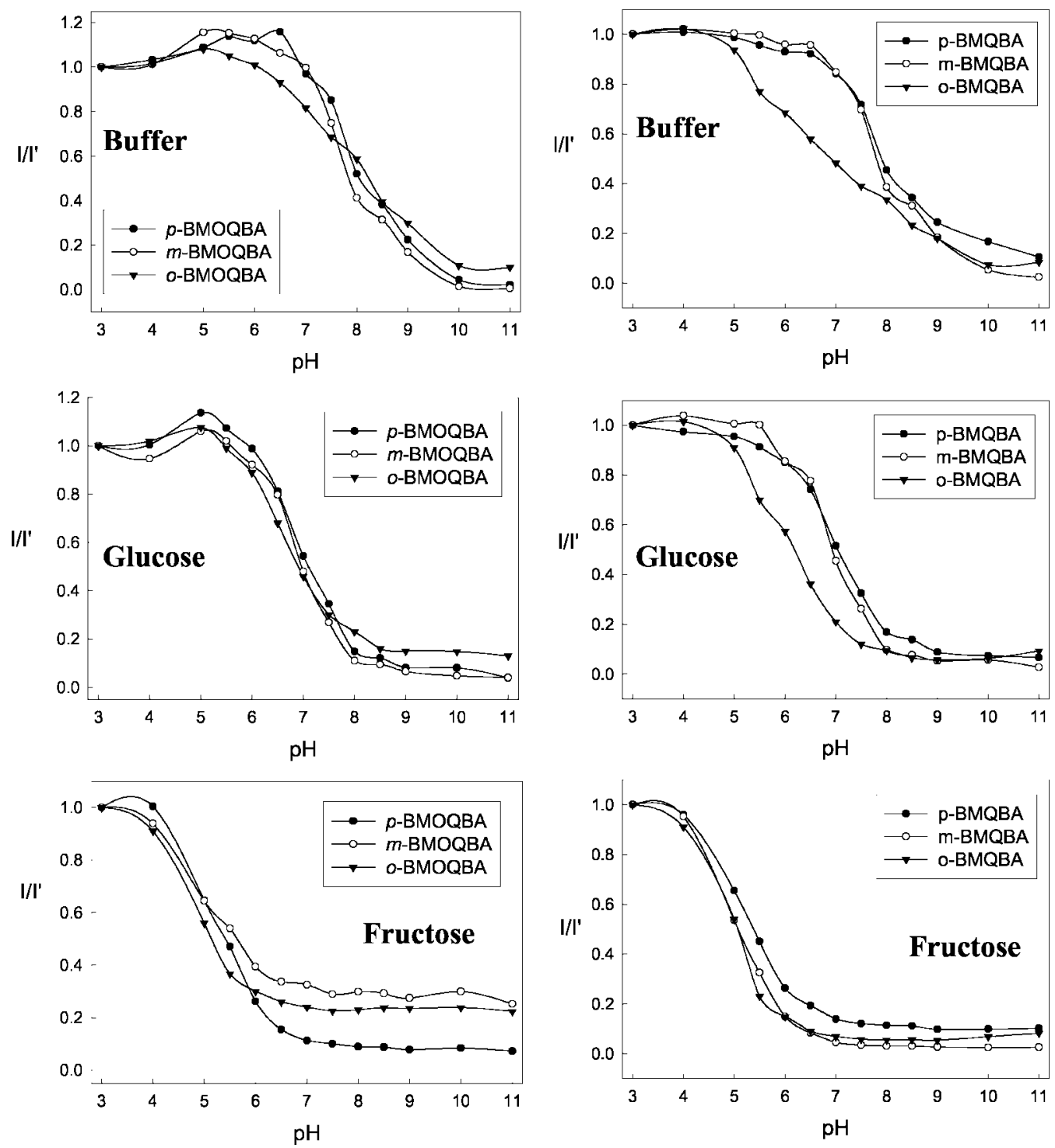

Fig. 8. The ratio of the emission intensities at band maximum as a function of $\mathrm{pH}$ for BMOQBAs (Top left) and BMQBAs (Top Right) and with $100 \mathrm{mM}$ Glucose (Middle left and Middle right) and $100 \mathrm{mM}$ Fructose (Bottom left and Bottom right) respectively.

Fig. 9. The boronic acid group is an electron-deficient Lewis acid having an $\mathrm{sp}^{2}$-hybridized boron atom with a triangular conformation. The anionic form of the boronic acid, formed in high $\mathrm{pH}$ solutions, is characterized by a more electron rich $\mathrm{sp}^{3}$-hybridized boron atom with a tetrahedral geometry. The change in the electronic properties and the geometry at the boron atom induces the fluorescence spectral changes of the probes. It is well-known that the quinine/quinoline compounds exhibit high quantum yields in acidic media, from the corresponding quaternized salt [46,47]. Similarly here, the boronic acid probes are more fluorescent in acidic solutions. However, when the $\mathrm{pH}$ of the medium is increased the electron density on the boron atom is increased, facilitating the partial neutralization of the positively charged quaternary nitrogen of the quinolinium moiety. We have termed this interaction as a charge neutralization-stabilization mechanism, and a schematic representation of this mechanism with regard to glucose binding/sensing is illustrated in Fig. 9. In contrast, the control compounds BMQ and BMOQ are unperturbed.

The Monosaccharide induced spectral changes of the probes are shown in Fig. 10. In an analogous manner to that described for increasing $\mathrm{pH}$ above, we observed 
Table II. $\mathrm{p} K_{\mathrm{a}}$ Values for $o-, m$ - and $p$-BMOQBA and -BMQBAs in Buffer and $100 \mathrm{mM}$ Sugars

\begin{tabular}{lccc}
\hline \multicolumn{1}{c}{ Medium } & $o$-BMOQBA & $m$-BMOQBA & $p$-BMOQBA \\
\hline \multirow{2}{*}{$\begin{array}{l}\text { Buffer } \\
\quad+100 \mathrm{mM} \text { Glucose }\end{array}$} & 6.90 & 7.70 & 7.90 \\
$\quad+100 \mathrm{mM}$ Fructose & 4.80 & 6.90 & 6.90 \\
& $o$-BMQBA & $m$-BMQBA & $p$-BMOQBA \\
\hline & 6.70 & 7.75 & 7.80 \\
Buffer & & & \\
$\quad+100 \mathrm{mM}$ Glucose & 6.10 & 6.85 & 6.95 \\
$\quad+100 \mathrm{mM}$ Fructose & 5.00 & 5.05 & 5.45 \\
\hline
\end{tabular}

a systematic decrease in fluorescence intensity of the boronic acid containing probes in $\mathrm{pH} 7.5$ phosphate buffer, for increasing glucose concentrations. The corresponding titration curves obtained by plotting $I^{\prime}$ divided by $I$, where $I^{\prime}$ and $I$ are fluorescence intensities at $427 \mathrm{~nm}$ for BMQBA and $450 \mathrm{~nm}$ for BMOQBA, in the absence and presence of sugar respectively, versus glucose concentration, are also shown in Fig. 11. The right-hand column of Fig. 11 shows the response of the probes in the tear glucose concentration range.

For the BMOQBA probes we typically see a greater response for the para- isomer, with a 2.4-fold change in signal for $50 \mathrm{mM}$ glucose. Interestingly, a $\approx 13 \%$ change in signal is observed up to $2 \mathrm{mM}$ glucose, noting that glucose levels in tears can change from $\approx 500 \mu \mathrm{M}$ for a healthy person, up to $5 \mathrm{mM}$ for diabetics. For ortho$\mathrm{BMQBA}$, then $\mathrm{a} \approx 16 \%$ change in fluorescence signal can be observed over a similar glucose range. Interestingly, for both classes of probes, the para-isomers show the weakest response towards glucose (Fig. 11). Given that the through-bond mechanism is expected to be prevalent for the para-isomers, and the ortho-isomer is expected to show both through bound and through space interac-

Table III. Dissociation Constants, $K_{\mathrm{D}}(\mathrm{mM})$, of the Probes with Glucose and Fructose in Buffer and in the Contact Lens

\begin{tabular}{|c|c|c|c|c|}
\hline \multirow[b]{2}{*}{ Probe } & \multicolumn{2}{|c|}{ Glucose } & \multicolumn{2}{|c|}{ Fructose } \\
\hline & Buffer & Lens & Buffer & Lens \\
\hline$o$-BMOQBA & 49.5 & 322.6 & 0.65 & 84.7 \\
\hline$M$-BMOQBA & 1000 & 54.6 & 1.8 & 4.9 \\
\hline$P$-BMOQBA & 430.0 & 111.1 & 9.1 & 34.7 \\
\hline$o$-BMOQBA & 100 & 17.9 & 4.7 & 34.8 \\
\hline$M$-BMOQBA & 476 & 58.1 & 13.2 & 21.6 \\
\hline$P$-BMOQBA & 370 & 128.2 & 13.8 & 12.9 \\
\hline
\end{tabular}

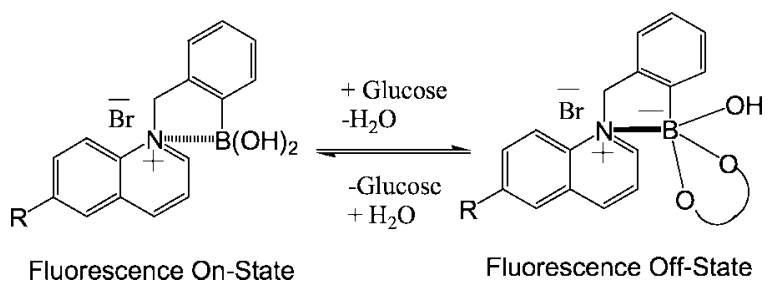

Fig. 9. A schematic representation of the charge neutralization-stabilization mechanism with regard to glucose sensing.

tions, then a greater response was indeed expected, as is observed, for the ortho-isomer. However, the much weaker response of the para-isomer suggests that steric hindrance, with regard to glucose binding, is not present here.

The dissociation constants of the probes with both glucose and fructose in $\mathrm{pH} 7.5$ phosphate buffer are presented in Table III, calculated using Eq. 2. As expected, a higher affinity for fructose is observed (lower $K_{\mathrm{D}}$ value), which is a general observation for monophenyl boronic acid derivatives [31-36], but it should be noted that the concentration of fructose in tears is substantially lower than for glucose [14-17]. A comparison of the trends in glucose response observed in Fig. 11, and the recovered $K_{\mathrm{D}}$ values in Table III, show some differences, which we have attributed to the difficulties encountered during data fitting. While beyond the scope of this text, these fitting difficulties clearly reflect the need for a new kinetic sugar binding function with our new probes. Further studies are underway in this regard.

\section{Response of the Glucose Signaling Probes in the Contact Lens}

Doped contact lenses, which were previously washed and allowed to leach dye for $1 \mathrm{hr}$ were tested with both glucose and fructose. Buffered solutions of sugars were added to the lens, $\mathrm{pH} 7.5$ phosphate buffer, in an analogous manner to ocular conditions. Fluorescence spectra were typically taken 15 min after each sugar addition to allow the lens to reach equilibrium. The $90 \%$ response time, the time for the fluorescence signal to change by $90 \%$ of the initial value, was $\approx 10 \mathrm{~min}$.

Figure 12 shows the response of $o$-BMOQBA and $o$-BMQBA, Top and Bottom left respectively, for increasing concentrations of glucose injected into the $1.5 \mathrm{~cm}^{3}$ contact lens volume. Similar to the solution based measurements, the probes show a decrease in fluorescence intensity, which we attribute to the complexation of glucose with boronic acid and the subsequent charge neutralization 

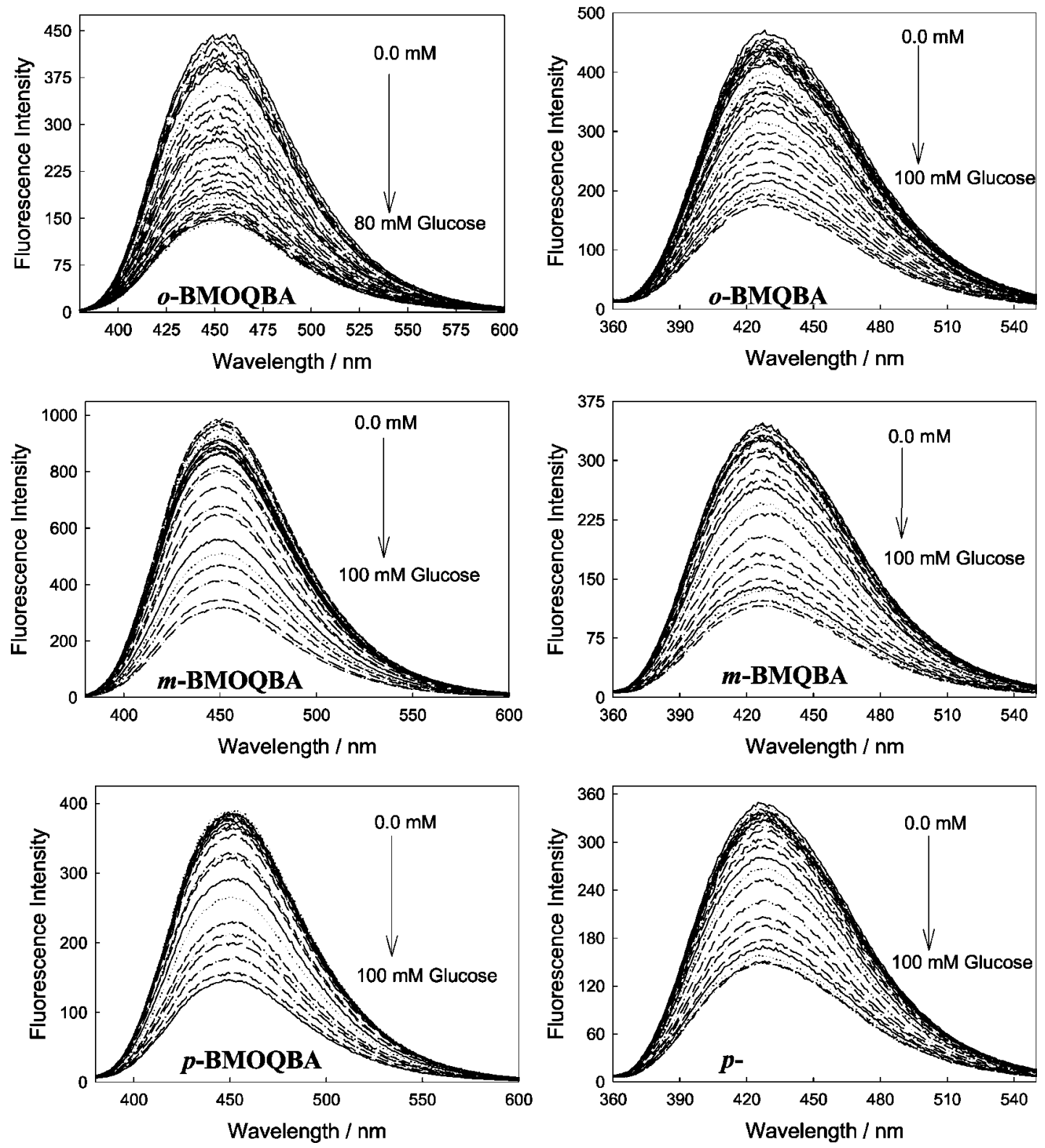

Fig. 10. Emission spectra of BMOQBAs (Left column) and BMQBAs (Right column) in pH 7.5 phosphate buffer with increasing glucose concentration. The $l_{\mathrm{ex}}$ for BMOQBAs was $345 \mathrm{~nm}$ and for BMQBAs $320 \mathrm{~nm}$.

mechanism described earlier. We were again able to construct the $I^{\prime} / I$ plots, where $I^{\prime}$ is the intensity in the absence of sugar (Fig. 12 right). As was observed in solution, Fructose had a greater response, reflecting the greater affinity of mono phenyl boronic acid derivatives for fructose. However, in the low sugar concentration ranges, $<2 \mathrm{mM}$ sugar, the response towards both sugars was comparable [15-17]. Differences in the response towards glucose for the isomers could also be observed in the lenses (Fig. 13), where $m$-BMOQBA was found to have a greatest response amongst this class of probes. From Fig. 13 we can clearly we see a greater response in lens towards sugars than in our solution based studies at $\mathrm{pH} 7.5$, with $p$-BMQBA showing a greater than $20 \%$ fluorescence signal change with as little as $2 \mathrm{mM}$ glucose. This wasn't unexpected, and is simply explained by the $\mathrm{p} K_{\mathrm{a}}$ of the probes being $<27$, the probes being compatible with the mildly acidic environment. Figure 14 directly compares the response of the probes in both contact lens and buffer, where we see a comparable if not better response towards glucose in 

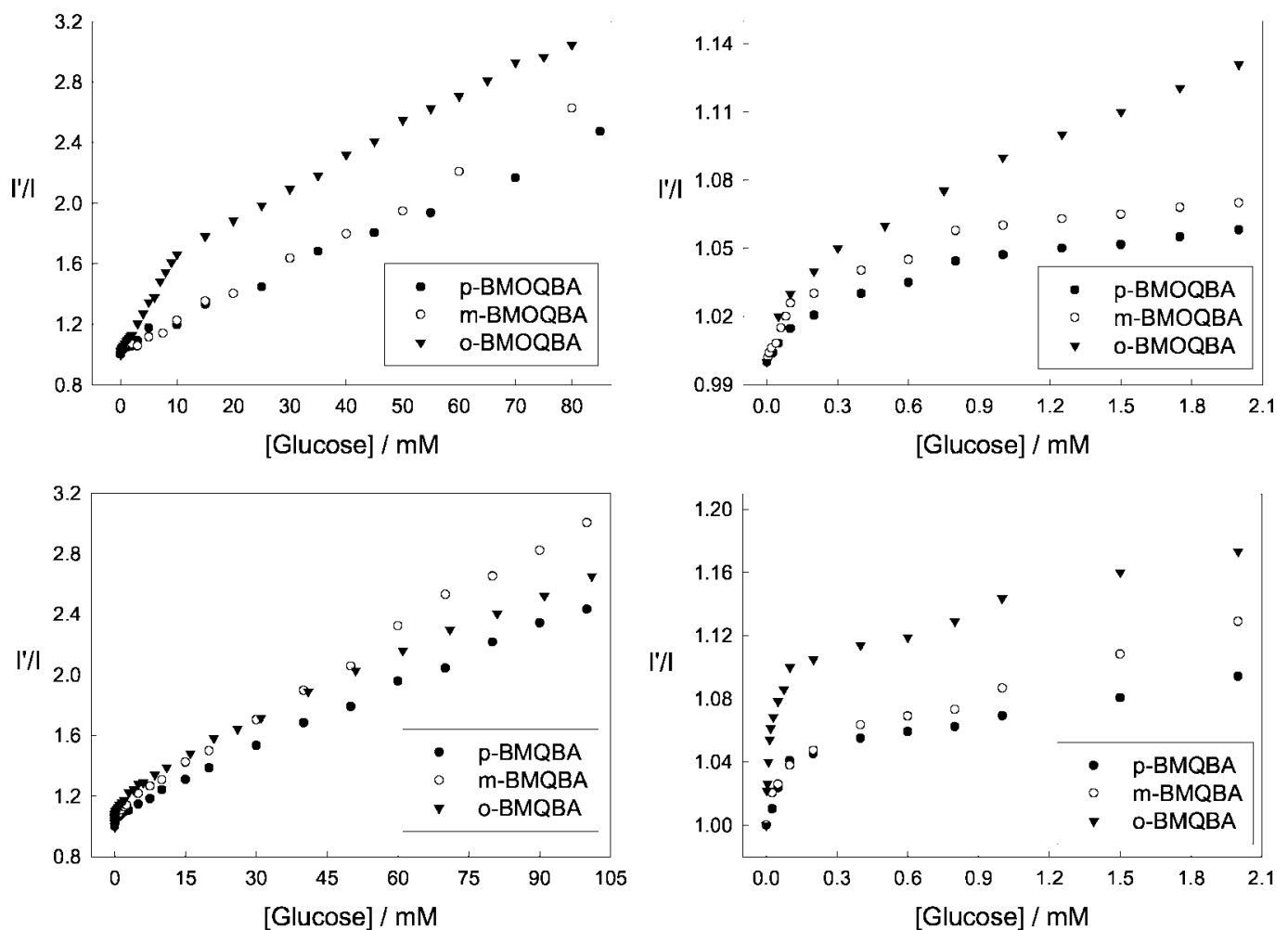

Fig. 11. Respective intensity ratio for all three isomers of BMOQBAs in the absence, $I^{\prime}$, and in the presence, $I$, of glucose, (Top left), and in the tear glucose concentration range (Top right). The corresponding plots for BMQBAs are also shown in the blood glucose concentration range and tear glucose concentration range (Bottom right and Bottom, respectively).

the lens. We are uncertain at this time however, as to the nature of the smaller fluorescence response at higher glucose concentrations in the lens, but we speculate that it may be due to the greater leaching of the glucose-bound (boronate diester) form of the probes and/or their displaced solubility within the contact lens polymer. This may also account for some of the complex binding kinetics we have observed, evident in some $I^{\prime} / \mathrm{I}$ Vs [sugar] plots. In this regard the response of doped lenses also show complex behavior towards $\mathrm{mM}$ fructose concentrations, with much simpler kinetics observed in the tear glucose concentration range (data not shown).

For all our glucose contact lens sensing studies we repeated these doped lens experiments several times, and in all cases the trends were reproducible. It is difficult to assess the effect of the PVA hydroxyl groups of the contact lens polymer on the response of boronic acid to sugar, but our studies with solutions of glycerol indicated that sugar had much higher binding affinities than glycerol hydroxyl groups. We therefore speculate that sugars will preferentially bind boronic acid groups in the PVA lens polymer. In any event the boronic acid probes function well (reversibly) towards sugars in the lens, in what is likely to be an environment saturated with PVA hydroxyl groups.

\section{Probe Leaching, Interferents, and Shelf Life}

Leaching studies of the probes from the contact lens polymer were undertaken using the lens holder, which contained $\approx 1.5 \mathrm{~cm}^{3}$ buffer, $20^{\circ} \mathrm{C}$. A Varian fluorometer measured the intensity change as a function of time to determine the percentage signal change, corresponding to dye leaching. It should be noted that with no sample present, no intensity fluctuations or drifts were observed, indicating stability of the fluorometer Xenon-arc source.

We were able to observe up to an $8 \%$ change in fluorescence intensity, attributed due to dye leaching, for the BMOQBA class of probes, with very little change after about 25 min (Fig. 15). In contrast, the BMQBA probes show a much greater extent of leaching over the same time period and under identical conditions. Given that the BMQBA probes typically showed a greater response towards glucose in the lens, then this suggests that the BMQBA probes may be more accessible in the lens, than 

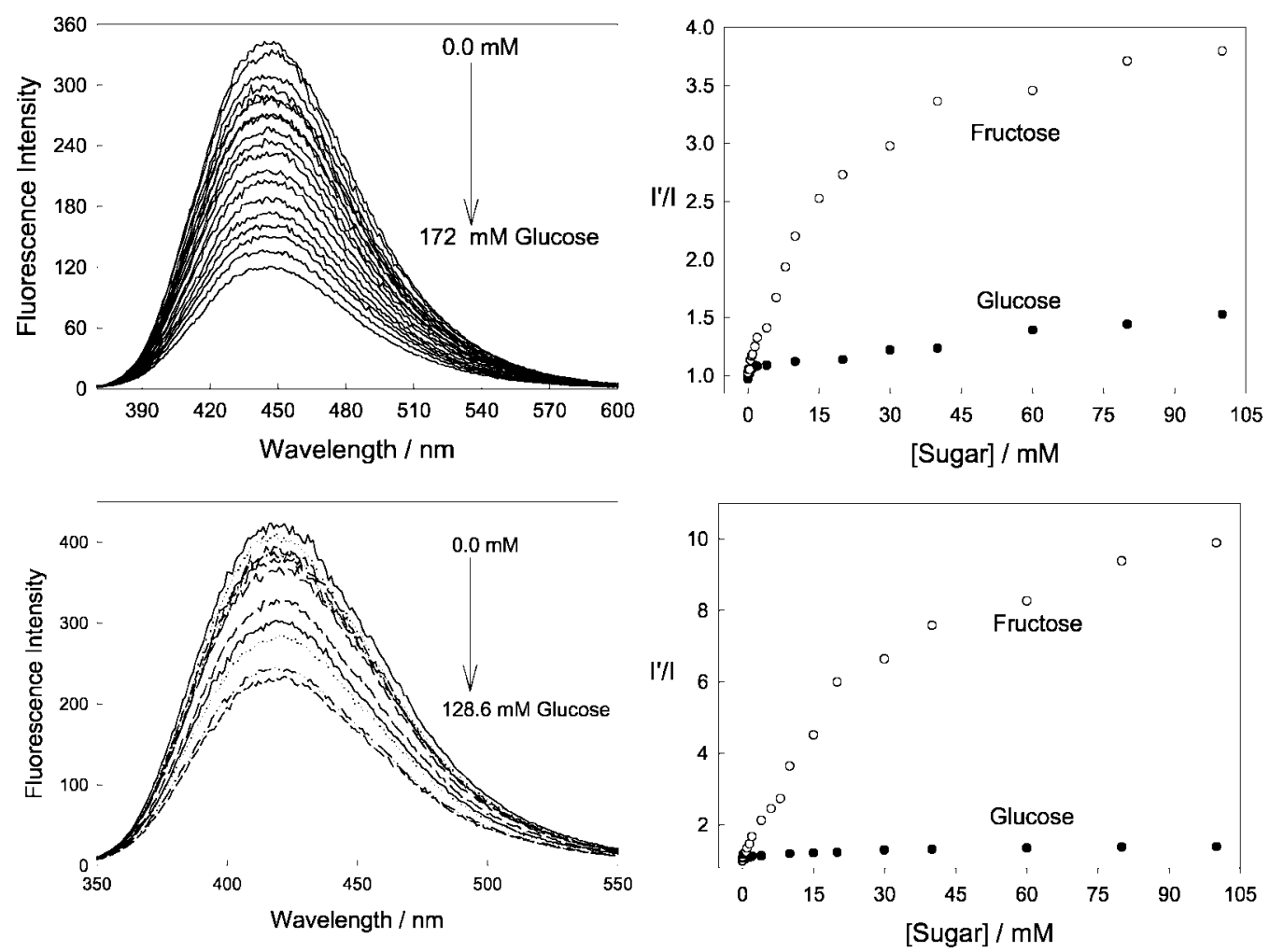

Fig. 12. The emission spectra of an $o-\mathrm{BMOQBA}$ and $o-\mathrm{BMQBA}$ doped contact lens in the presence of increasing glucose concentrations (Top left and Bottom left, respectively). $\lambda_{\mathrm{ex}}=345 \mathrm{~nm}$ for $o$-BMOQBA and $320 \mathrm{~nm}$ for $o$-BMQBA. The corresponding emission intensity ratio at band maximum for $o$-BMOQBA and $o$-BMQBA doped contact lens in the absence, $I^{\prime}$, and presence, $I$, of both Glucose and Fructose (Top right and Bottom right, respectively).

the BMOQBA probes. In addition, similar results were obtained at $38^{\circ} \mathrm{C}$ but with a different leaching rate.

In all our lens response studies described here, lenses were pre-leached to a steady-state fluorescence intensity before use. After glucose measurements were undertaken the outer lens fluid volume surrounding the contact lens was found to be non-fluorescent indicating that dye had not leached from the lens during actual glucose sensing measurements. It should be noted that while chemistries are available to covalently label our probes within the contact lens polymer, which would eliminate any leaching, it is an important design concern for our approach that the lenses remain unmodified, so that their physiological characteristics and compatibilities remain unchanged. In fact our approach is targeted at reducing future lens redesign costs for industry, by using simple probe doping.

As with all sensors it is important to consider the effects of potential interferents and sensor shelf-life on the working response of the device. Throughout much of this paper we have shown the response of the probes towards fructose, primarily because of its well-known greater affinity for the boronic acid moiety [31-36]. However, the con- centration of fructose in blood is $\approx 10$ times lower than glucose [14], a relationship which is also thought to occur in tears [14]. Hence fructose is not thought to be a major interferent in tears, simply shown here to place the binding trends in context. However, tears are a complex mixture of proteins and other analtyes, such as sodium (120$170 \mathrm{mM})$, potassium $(6-26 \mathrm{mM})$ and chloride $(100 \mathrm{mM})$ [50]. We subsequently tested these new contact lens probes with various aqueous halides, given that $\mathrm{Na}^{+}$and $\mathrm{K}^{+}$ are unlikely to perturb our fluorophores (Table IV). As expected, the BMOQBA probes are modestly quenched by chloride, with steady-state Stern-Volmer constants in the range $170-182 \mathrm{M}^{-1}$, the BMQBA probes having significantly smaller quenching constants in the range 17$44 \mathrm{M}^{-1}$. This result is simply explained by the shorter lifetime of the BMQBA probes as compared to the BMOQBA probes, and the probability of an excited-state chloride ion encounter $[41,45]$. Encouragingly, the BMQBA probes typically showed a greater response towards glucose as well as being the least perturbed by aqueous chloride. In any event, simple corrections in the fluorescence signal can readily account for chloride interference on the 

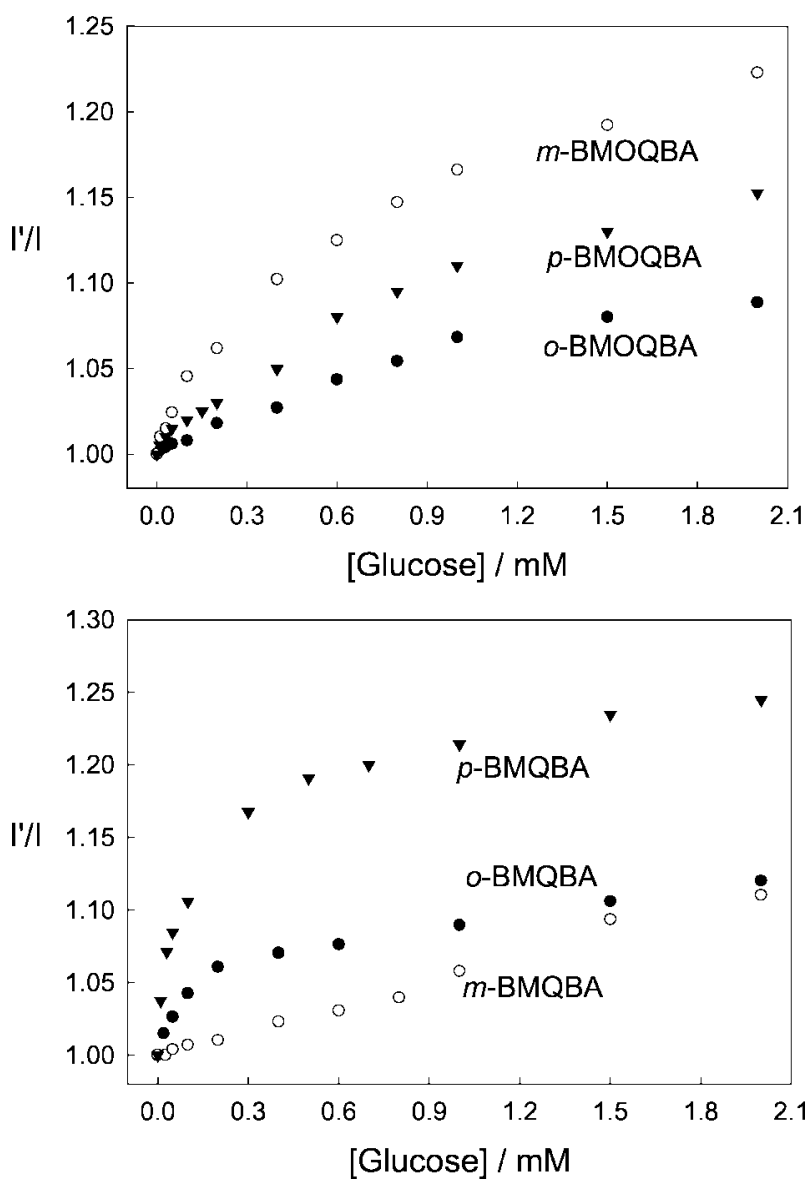

Fig. 13. The response of BMOQBAs (Top) and BMQBAs (Bottom) in the contact lens in the tear glucose concentration range.

glucose response. This is shown in Fig. 2. Sensors spots on the surface of the lens could contain a reference chloride compound or indeed another probe sensitive to both glucose and chloride. By employing the extended SternVolmer equation for multiple analytes [45], one can easily correct for background interferences.

It is also informative to consider the $\mathrm{pH}$ of tears as a potential interferent, given the response of these probes to $\mathrm{pH}$ as shown in Fig. 8. It is known that unstimulated tear $\mathrm{pH}$ levels can vary in the range 7.14-7.82 measured from healthy subjects at different times of the day, with a typical mean value around $\mathrm{pH} 7.45$ [50]. However, a more acidic $\mathrm{pH}$ of less than 7.3 is found following prolonged lid closure, e.g. after sleep, which is thought to result from carbon dioxide produced by the cornea and trapped in the tear pool under the eye lids. While solutions of these new probes would be susceptible to these changes in $\mathrm{pH}$, we have found that the doped lenses we studied were indeed unbufferable, hence external changes in $\mathrm{pH}$ are most unlikely to affect the glucose response.
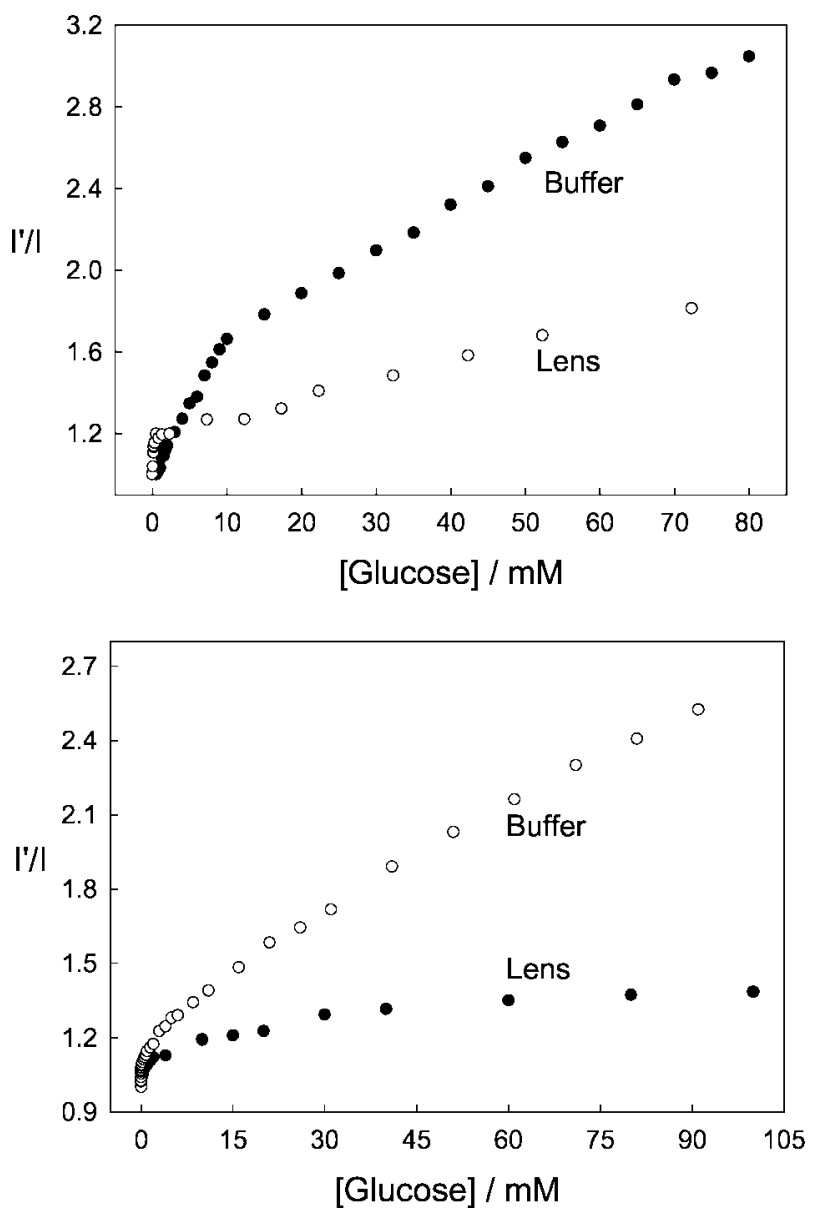

Fig. 14. A comparison of the emission intensity ratio for the $o$-BMOQBA doped contact lens with that obtained in $\mathrm{pH} 7.5$ phosphate buffer, in the absence, $I^{\prime}$, and presence, $I$, of Glucose (Top) and the corresponding plot for $o$-BMQBA (Bottom).

With regard to the glucose-sensing contact lens shelflife, lenses that had been doped, leached and stored for several months both wet and dry, gave identical sugar sensing results, indicating no lens polymer-fluorophore interactions over this time period, or indeed probe degradation.

\section{FUTURE DEVELOPMENTS BASED ON THIS TECHNOLOGY}

\section{Continuous and Non-Invasive Glucose Monitoring}

In this review article of work from our laboratories, we have shown that fluorescent probes can be fabricated to be compatible with commercially available contact lenses, which have already been assessed and optimized with regard to vision correction and oxygen permeability. This has enabled the first prototype based on this new approach 

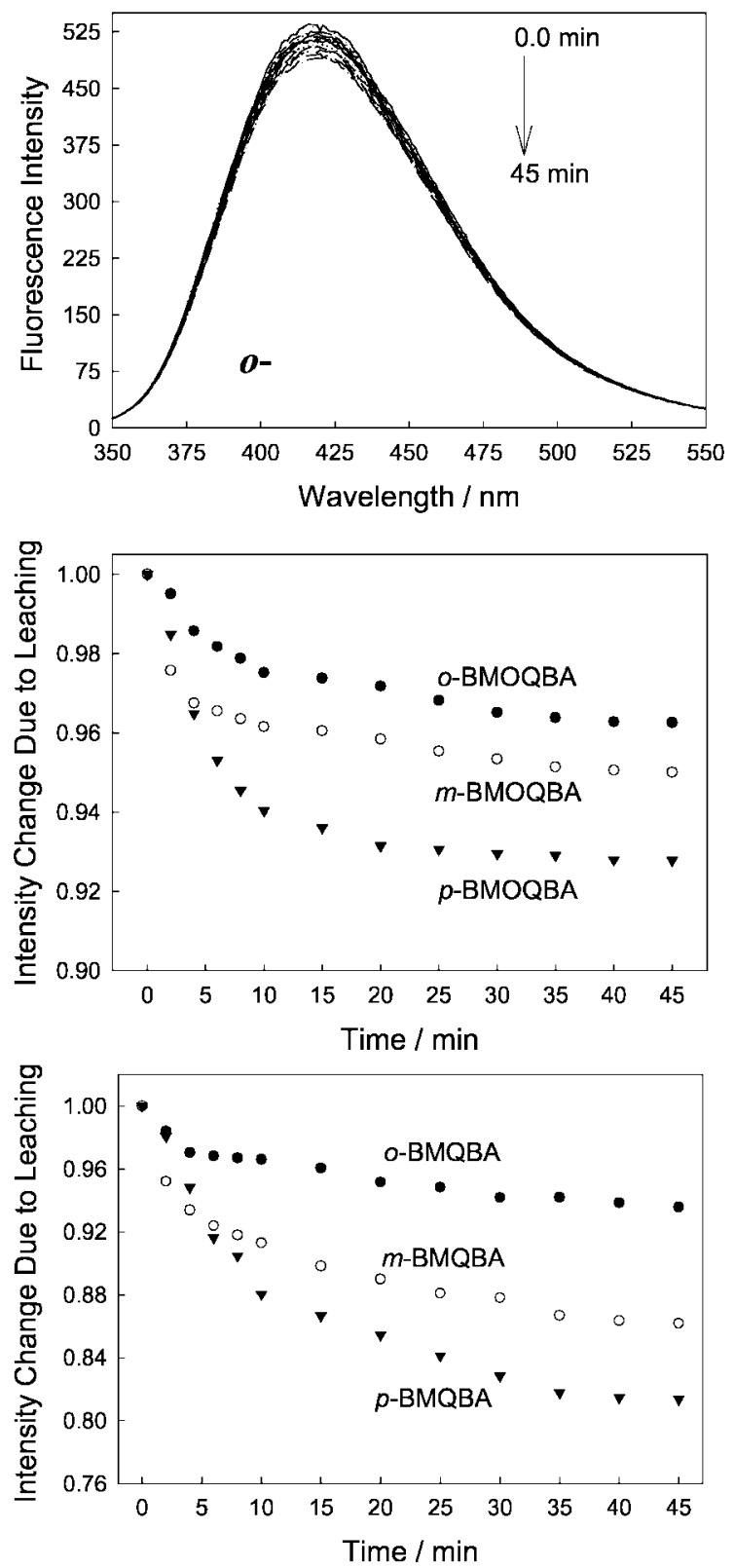

Fig. 15. Emission spectra of $o$-BMQBA in the lens immersed in $\mathrm{pH} 7.5$ buffer with time (Top). $l_{\mathrm{ex}}=320 \mathrm{~nm}$. Normalized intensity change at band maximum of BMOQBA with time due to leaching (Middle), and that for the BMQBAs (Bottom).

to be realized. With regard to glucose monitoring by this approach, we speculate on several future improvements to this technology.

Clear or colored contact lenses. Many boronic containing fluorophores have a visible absorption [31-36], which apart from their lack of glucose sensitivity in the lens as discussed earlier [14], would introduce color into
Table IV. Stern-Volmer Constants of the Probes with Halides in Water

\begin{tabular}{lccc}
\hline \multicolumn{1}{c}{ Probe } & $\mathrm{CI}^{-}\left(K_{\mathrm{sv}} / \mathrm{M}^{-1}\right)$ & $\mathrm{Br}^{-}\left(K_{\mathrm{sv}} / \mathrm{M}^{-1}\right)$ & $\mathrm{I}^{-}\left(K_{\mathrm{sv}} / \mathrm{M}^{-1}\right)$ \\
\hline$o$-BMOQBA & 170 & $332^{a}$ & $471^{\mathrm{a}}$ \\
$M$-BMOQBA & 182 & 413 & 540 \\
$P$-BMOQBA & 177 & 370 & 959 \\
BMOQB & 222 & 384 & 520 \\
$o$-BMQBA & 44.0 & 55.0 & 97.0 \\
$M$-BMQBA & 20.0 & 32.0 & 48.0 \\
$P$-BMQBA & 17.0 & 26.5 & 42.0 \\
BMQB & 35.0 & 55.0 & 71.0 \\
\hline
\end{tabular}

aThe concentrations of $\mathrm{Br}^{-}$and $\mathrm{I}^{-}$in tears is extremely low and is unlikely to be an interferent in our glucose measurements.

a doped lens. While colored lenses are attractive to a few people as sports or even fashion accessories, the majority of contact lenses worn today are clear, hence our colorless lenses described here are ideal in this regard. One disadvantage however with our lenses is the requirement for an excitation and detection device as shown in Fig. 2. One improvement to our technology could be the use of colored contact lenses, which change color due to the concentration of tear and therefore blood glucose. This can be achieved by the ground-state binding of glucose to boronic acid and the subsequent changes in fluorophore absorption spectrum. A patient wearing the lenses could simply look in the mirror, or the color even determined by an on-looker, and compared to a precalibrated color strip, one could assess the extent of hyperglycemia. This technology would be most attractive to parents of young diabetic children or for care workers of the elderly. Work in currently underway in our laboratories in this regard.

Sensor spots or doped lenses. As briefly mentioned earlier and shown in Fig. 2, sensor spots on the surface of contact lenses could correct signal responses for interferents such as aqueous chloride. Indeed the spots could either be visible to the wearer (self-readout) or readable by an external monitoring device.

Detection methods. While simple colorimetric methods are likely to be the most simple to introduce to the market place, other fluorescence sensing methodologies, such as polarization, lifetime and ratiometric sensing, offer many spectroscopic sensing advantages over the simple intensity measurements described for our lenses [41]. For example, fluorescence lifetime and ratiometric measurements are independent on total light intensity or indeed fluctuations in ambient room light.

\section{Clinical Condition and Diagnosis from Tears}

Given that tear glucose can be continuously and noninvasively monitored using our lens approach, then it may 
be possible for both clinical condition assessment and disease diagnosis using the contact lens sensing platform, and suitably designed fluorophores. In fact, as compared to saliva, tears represent a more stable body fluid of low protein concentration and with only modest variations in $\mathrm{pH}$.

For example, for clinical condition assessment, $\mathrm{Na}^{+}$, $\mathrm{K}^{+}, \mathrm{Ca}^{2+}, \mathrm{Mg}^{2+}, \mathrm{pH}$, Histamine, Urea, Lactate, Cholesterol and Glucose in tears are known to directly track or relate to the serum levels [50]. Indeed, one could potentially even track body core temperature using thermochromic type probes [51], embedded within contact lenses or even sensor spots.

For disease diagnosis, the possibility of diagnosing; Glaucoma, Sjogrens disease, Lysosomal storage diseases, corneal ulceration and bacterial infections could be realized by designing lenses to detect; Catecholamines, Lysozyme, Lysosomal enzymes, Collagenase and $\alpha$ Antitrypsin respectively. It is possible that many other clinical conditions and diseases could also be either monitored or diagnosed via this approach, although relatively little tear biochemistry is known [50].

\section{Drug Testing, Compliance, and Screening}

Saliva is and has been used for therapeutic drug monitoring, by predicting the free fraction of drugs in blood from that determined in saliva. Changes in the free drug levels can have important clinical consequences, since either toxic or sub-therapeutic levels may exist, even when the total drug concentration is in the normal range. Coupled with the fact that saliva concentrations varying greatly in both $\mathrm{pH}$ and composition, obviates the need for a novel clinical sensing platform for drug testing, compliance and screening. As mentioned earlier, tear fluid has a relatively lower protein concentration with only slight changes in $\mathrm{pH}$, where the passage of drugs from plasma to tears takes place by diffusion of the non-protein bound fraction [50]. Subsequently, tears have already been used to assess the concentration of antibiotics such as Ampicillin and the Anticonvulsants, Phenobarbital and Carbamazepine [50]. However, these drug concentration measurements are inherently difficult due to the $5-10 \mu \mathrm{L}$ total tear volume to be sampled [50]. Indeed, while tear glucose levels have been known to be elevated during hyperglycemia for nearly 70 years, it is the difficulties associated with tear collection, which has limited the practical use of tears for diabetes mellitus assessment. However, similar to our glucose sensing contact lenses, it may be possible to develop lenses for drug testing, based on either colorimetric or other fluorescence spectroscopic based methodologies.

\section{CONCLUSIONS}

We have developed a range of new glucose sensing contact lenses, by doping strategically designed fluorescent probes into commercially available contact lenses. The probes are completely compatible with the new lenses and can readily detect glucose changes up to several $\mathrm{mM}$ glucose, appropriate for the tear glucose concentration range for diabetics, i.e. $\approx 500 \mu \mathrm{M} \rightarrow 5 \mathrm{mM}$ [14].

The lenses have a $90 \%$ response time of about $10 \mathrm{~min}$, allowing the continuous and noninvasive monitoring of ocular glucose. This is a significant improvement over enzymatic methods based on blood sampling by finger pricking, with many diabetics begrudgingly testing between 4 and 6 times daily.

With diabetes being widely recognized as one of the leading causes of death and disability in the western world, we believe our boronic acid doped contact lens approach and findings, are a notable step forward towards the continuous and non-invasive monitoring of physiological glucose.

\section{ACKNOWLEDGMENTS}

The authors would like to thank the University of Maryland Biotechnology Institute and the NIH, National Center for Research Resources, RR-08119, for financial support. Drs Angelika Domschke and Dawn Smith of Cibavision are also acknowledged for supplying the contact lens holder shown in Fig. 6.

\section{REFERENCES}

1. Leonid Poretsky (Ed.) (2002). Principles of Diabetes Mellitus, Kluwer Academic/Plenum, Norwell, MA.

2. V. C. Medvei (1993). In The History of Clinical Endocrinology: A Comprehensive Account of Endocrinology from Earliest Times to Present Day, Parthenon, New York.

3. M. R. Robinson, R. P. Eaton, D. M. Haaland, G. W. Koepp, E. V. Thomas, B. R. Stallard, and P. L. Robinson (1992). Non-invasive glucose monitoring in diabetic patients: A preliminary evaluation. Clin. Chem. 38, 1618-1622.

4. H. M. Heise, R. Marbach, T. H. Koschinsky, and F. A. Gries (1994). Non-invasive blood glucose sensors based on near-infrared spectroscopy. Ann. Occup. Hyg. 18, 439-447.

5. W. F. March, B. Rabinovitch, R. Adams, J. R. Wise, and M. Melton (1982). Ocular Glucose sensor. Trans. Am. Soc. Artif. Intern. Organs 28, 232-235.

6. B. Rabinovitch, W. F. March, and R. L. Adams (1982). Non-invasive glucose monitoring of the aqueous humor of the eye, Part 1: Measurement of very small optical rotations. Diabetes Care 5, 254-258.

7. G. M. Schier, R. G. Moses, I. E. T. Gan, and S. C. Blair (1988). An evaluation and comparison of reflolux Iiand Glucometer II, two new portable reflectance meters for capillary blood glucose determination. Diabetes Res. Clin. Pract. 4, 177-181.

8. W. Clarke, D. J. Becker, D. Cox, J. V. Santiago, N. H. White, J. Betschart, K. Eckenrode, L. A. Levandoski, E. A. Prusinki, L. M. Simineiro, A. L. Snyder, A. M. Tideman, and T. Yaegar (1988). 
Evaluation of a new system for self blood glucose monitoring. Diabetes Res. Clin. Pract. 4, 209-214.

9. W. Trettnak and O. S. Wolfbeis (1989). Fully reversible fiberoptic glucose biosensor based on the intrinsic fluorescence of glucose-oxidase. Anal. Chim. Acta 221, 195-203.

10. D. Meadows and J. S. Schultz (1988). Fiber optic biosensor based on fluorescence energy transfer. Talanta 35, 145-150.

11. L. Tolosa, H. Malak, G. Rao, and J. R. Lakowicz (1997). Optical assay for glucose based on the luminescence decay time of the long wavelength dye Cy5. Sens. Actuators B 45, 93-99.

12. L. Tolosa, I. Gryczynski, L. R. Eichorn, J. D. Dattelbaum, F. N. Castellano, G. Rao, and J. R. Lakowicz (1999). Glucose sensors for low cost lifetime-based sensing using a genetically engineered protein. Anal. Biochem. 267, 114-120.

13. S. D'Auria, N. Dicesare, Z. Gryczynski, I. Gryczynski, M. Rossi, and J. R. Lakowicz (2000). A thermophilic apoglucose dehydrogenase as a nonconsuming glucose sensor. Biochem. Biophys. Res. Commun. 274, 727-731.

14. R. Badugu, J. R. Lakowicz, and C. D. Geddes (2004). The noninvasive continuous monitoring of physiological glucose using a novel monosaccharide-sensing contact lens. Anal. Chem. 76, 610-618.

15. R. Badugu, J. R. Lakowicz, and C. D. Geddes (2003). A glucose sensing contact lens: A non-invasive technique for continuous physiological glucose monitoring.J. Fluorescence 13, 371-374.

16. C. D. Geddes, R. Badugu, and J. R. Lakowicz (2004). Contact lenses may provide window to blood glucose. Biophotoincs int. February(2), 50-53.

17. R. Badugu, J. R. Lakowicz, and C. D. Geddes (2004). Ophthalmic glucose sensing: A novel monosaccharide sensing disposable and colorless contact lens. The Analyst 129, 516-521.

18. J. M. Sugihara and C. M. Bowman (1958). Cyclic benzeneboronate esters.J. Am. Chem. Soc. 80(10), 2443-2446.

19. J. P. Lorand and J. O. Edwards (1959). Polyol complexes and structure of the benzeneboronate ion. J. Org. Chem. 24(6), 769-774.

20. G. Springsteen and B. Wang (2002). A detailed examination of boronic acid-diol complexation. Tetrahedron 58(26), 5291-5300.

21. T. D. James, K. R. A. S. Sandanayake, and S. Shinkai (1995). Chiral discrimination of monosaccharides using a fluorescent molecular sensor. Nature 374, 345 .

22. J. C. Norrild and H. Eggert (1995). Evidence for monodentate and bidentate boronate complexes of glucose in the furanose form-Application of (1)J(C-C)-coupling-constants as a structural probe. J. Am. Chem. Soc. 117(5), 1479-1484.

23. H. Eggert, J. Frederiksen, C. Morin, and J. C. Norrild (1999). A new glucose-selective fluorescent bisboronic acid. First report of strong alpha-furanose complexation in aqueous solution at physiological pH. J. Org. Chem. 64(11), 3846-3852.

24. W. Yang, H. He, and D. G. Drueckhammer (2001). Computerguided design in molecular recognition: Design and synthesis of a glucopyranose receptor. Angew. Chem. Int. Ed. 40(9), 1714-1718.

25. W. Wang, S. Gao, and B. Wang (1999). Building fluorescent sensors by template polymerization: The preparation of a fluorescent sensor for D-fructose. Org. Lett., 1(8), 1209-1212.

26. S. Gao, W. Wang, and B. Wang (2001). Building fluorescent sensors for carbohydrates using template-directed polymerizations. Bioorg. Chem. 29, 308-320.

27. J. J. Lavigne and E. V. Anslyn (1999). Teaching old indicators new tricks: A colorimetric chemosensing ensemble for tartrate/malate in beverages. Angew. Chem. Int. Ed. 38(24), 3666-3669.

28. J. Yoon and A. W. Czarnik (1992). Fluorescent chemosensors of carbohydrates. A means of chemically communicating the binding of polyols in water based on chelation-enhanced quenching. J. Am. Chem. Soc. 114, 5874-5875.

29. B. D. Smith, S. J. Gardiner, T. A. Munro, M. F. Paugam, and J. A. Riggs (1998). Facilitated transport of carbohydrates, catecholamines, and amino acids through liquid and plasticized organic membranes. J. Incl. Phenom. Mol. Recogn. Chem. 32, 121-131.
30. S. Soundararajan, M. Badawi, C. M. Kohlrust, and J. H. Hagerman (1989). Boronic acids for affinity-chromatography-spectral methods for determinations of ionization and diol-binding constants. Anal. Biochem. 178, 125-134.

31. T. D. James, K. R. A. S., and S. Shinkai (1994). A glucose-selective molecular fluorescence sensor. Angew, Chem. Int. Ed. 33(21), 2207-2209.

32. T. D. James, K. R. A. S. Sandanayake, R. Iguchi, and S. Shinkai (1995). Novel saccharide-photoinduced electron-transfer sensors based on the interaction of boronic acid and amine. J. Am. Chem. Soc. 117(35), 8982-8987.

33. N. Dicesare and J. R. Lakowicz (2001). Evaluation of two synthetic glucose probes for fluorescence-lifetime based sensing. Anal. Biochem. 294, 154-160.

34. N. Dicesare and J. R. Lakowicz (2001). Wavelength-ratiometric probes for saccharides based on donor-acceptor diphenylpolyenes. J. Photochem. Photobiol. A: Chem. 143, 39-47.

35. N. Dicesare and J. R. Lakowicz (2001). New color chemosensors for monosaccharides based on Azo dyes. Org. Lett. 3(24), 3891-3893.

36. N. Dicesare and J. R. Lakowicz (2002). Chalcone-analogue fluorescent probes for saccharides signaling using the boronic acid group. Tet. Lett. 43, 2615-2618.

37. V. V. Karnati, X. Gao, S. Gao, W. Yang, W. Ni, S. Sankar, and B. Wang (2002). A glucose-selective fluorescence sensor based on boronic acid-diol recognition. Bioorg. Med. Chem. Lett. 12, 3373-3377.

38. N. Dicesare and J. R. Lakowicz (2002). Charge transfer fluorescent probes using boronic acids for monosaccharide signaling. J. Biomed. Opt. 7(4), 538-545.

39. R. Badugu, J. R. Lakowicz, and C. D. Geddes (manuscript submitted for publication). Fluorescence sensors for monosaccharides based on the 6-methylquinolinium nucleus and boronic acid moiety: Application to ophthalmic diagnostics. Talanta.

40. R. Badugu, J. R. Lakowicz, and C. D. Geddes (manuscript submitted for publication). Boronic acid fluorescent sensors for monosaccharide signaling based on the 6-methoxyquinolinium heterocyclic nucleus: Progress towards noninvasive and continuous glucose monitoring. Bioorg. Med. Chem.

41. J. R. Lakowicz (1997). Principles of Fluorescence Spectroscopy 2nd ed., Kluwer/Academic Plenum, New York.

42. N. J. Turro, B. H. Baretz, and P. I. Kuo (1984). Photoluminescence probes for the investigation of interactions between sodium dodecylsulfate and water-soluble polymers. Macromolecules 17(7), 13211324.

43. K. Kalyanasundaram and J. K. Thomas (1977). Environmental effects on vibronic band intensities in pyrene monomer fluorescence and their application in studies of micellar systems. J. Am. Chem. Soc. 99(7), 2039-2044.

44. N. Dicesare and J. R. Lakowicz (2001). Spectral properties of fluorophores combining the boronic acid group with electron donor or withdrawing groups. Implication in the development of fluorescence probes for saccharides. J. Phys. Chem. A 105(28), 6834-6840.

45. C. D. Geddes (2001). Optical halide sensing using fluorescence quenching: Theory, simulations and applications-A review. Meas. Sci. Tech. 12(9), R53.

46. O. S. Wolfbeis and E. Urbano (1982). J. Heterocyclic Chem. 19, 841-843.

47. C. D. Geddes, K. Apperson, J. Karolin, and D. J. S. Birch (2001). Chloride sensitive probes for biological applications. Dyes Pigments 48, 227-231.

48. M. A. Fox and M. Chanon (Eds.) (1998). Photoinduced Electron Transfer Parts A-D, Elsevier, New York.

49. G. J. Kavarnos (1993). Fundamentals of Photoinduced Electron Transfer $\mathrm{VCH}, \mathrm{New}$ York.

50. N. J. Van Haeringen (1981). Climical biochemistry in tears. Survey Ophthalmol. 26(2), 84-96,

51. N. Chandrasekharan and L. Kelly (2004). in C. D. Geddes and J. R. Lakowicz (Eds.), Reviews in Fluorescence 2003, Kluwer Academic/Plenum, New York. 